\title{
WEAK INELASTIC PRODUCTION AND LEPTONIC DECAYS OF HEAVY LEPTONS ${ }^{\dagger}$
}

\author{
A. Soni \\ Columbia University, New York, N.Y. 10027
}

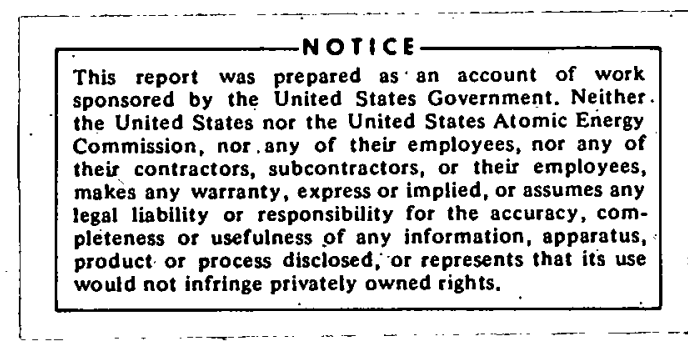

This research was supported in part by the U.S. Atomic Energy Commission. 


\section{DISCLAIMER}

This report was prepared as an account of work sponsored by an agency of the United States Government. Neither the United States Government nor any agency Thereof, nor any of their employees, makes any warranty, express or implied, or assumes any legal liability or responsibility for the accuracy, completeness, or usefulness of any information, apparatus, product, or process disclosed, or represents that its use would not infringe privately owned rights. Reference herein to any specific commercial product, process, or service by trade name, trademark, manufacturer, or otherwise does not necessarily constitute or imply its endorsement, recommendation, or favoring by the United States Government or any agency thereof. The views and opinions of authors expressed herein do not necessarily state or reflect those of the United States Government or any agency thereof. 


\section{DISCLAIMER}

Portions of this document may be illegible in electronic image products. Images are produced from the best available original document. 


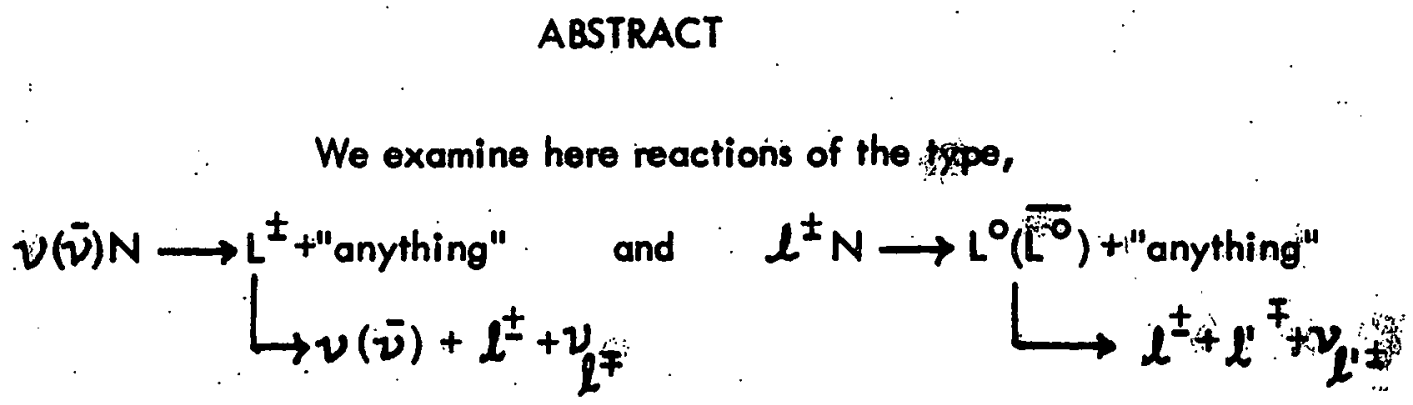

where $L$ is a lepton heavier than the muon, and derive expressions for the inclusive cross sections of the outgoing charged leptons. The phase space integration is simplified by negleting the electron and muon masses and the differential cross section, with respect to the transverse momentum of the charged leptons in the final state, are calculated. Using a simple. parameterization for the hadronic structure functions, results are presented for several incident lepton energies, between 50 and $500 \mathrm{GoV}$. in the lab frame, and,for heavy lepton masses ranging from 2 to $11 \mathrm{GeV}$, assuming the coupling constant of the heavy lepton to be identical to that of the muon. A simple relation between the differential cross section of the outgoing charged leptons along the beam axis and the total cross section is abtoined Throughout these calculdtions the effects produced on the spectrum of the final charged lepton by the polarization of the heavy lepton are fully incorporoted. 
1. Introduction

The existence of leptons, both charged and neutral, heavier than the muon has been predicted by several recent theories of weak interactions. A number of experiments ${ }^{2}$ for detection of these leptons are currently in progress. Since these particles are expected to have a very short lifetime $\left(\leqslant 10^{-11}\right.$ sec.) experimentally one has to look for their signatures in their decay products. The characteristic effect of such a heavy lepton on some aspects of the inclusive $l N$ spectrum has been examined by a number of authors, but effects due to polarization of the heavy lepton have been neglected 3,4 .

In this work we take into account the polarization of the heavy lepton produced weakly in $\ell N$ inelastic reactions and derive expressions for the cross section of the out going charged leptons. We simplify the phase space integration by neglecting the electron and muon masses and use a simple parameterization for the hadronic structure functions 5 . The differential cross section $\frac{d \sigma}{d Q_{t}^{2}}$, where $Q_{t}$ is component of the final charged lepton's momentum that is transverse to the direction of the incident lepton beam in the laboratory frame, is computed for heavy lepton masses of $2,5,8$ and $11 \mathrm{GeV}$, taking the incident lepton energy $E=50,100,200$ and 500 $\mathrm{GeV}$ and assuming that the coupling constant of the heavy lepton is identical to that of the muon. In the decay of a neutral heavy lepton where there are two charged leptons in the final state, we calculate the differential cross sections for both. Results are displayed graphically taking the incident beam to be $\mu^{-}, \mu^{+}$vor $\bar{\nu}$ and assuming that the heavy lepton couples via $V-A$ or $V+A$. These results are compared with a similar calculation where the polarization of the heavy lepton is ignored. 
We also show that as $Q_{t} \rightarrow 0$, the remaining variables of the outgoing lepton can be integrated analytically yielding a simple relation for $\left.\frac{d \sigma}{d Q_{t}^{2}}\right|_{Q_{t}=0}$

given by

$$
\left.\frac{d \sigma}{d Q_{t}^{2}}\right|_{Q_{t}=0}=\frac{8}{m_{L}^{2}} \sigma_{p} R
$$

where $\sigma_{p}$ is the cross section for production of heavy leptons of mass $m_{L}$ and $R$ is the branching ratio for the leptonic decay mode under consideration. 


\section{Calculations}

For definiteness, we concentrate on the reaction ${ }^{6}$ see Fig. 1):

$$
\begin{aligned}
v(k)+N(p) \rightarrow L^{-}\left(k^{\prime}\right)+\text { "anything" } & \\
& \longrightarrow v(K)+\ell^{-}(Q)+\bar{v}_{l}(\bar{k}) .
\end{aligned}
$$

where $L$ is a heavy lepton and $l$ may be e or $\mu$. In the limit when the electron and the muon masses, $m_{e}, m_{\mu} \rightarrow 0$, the expressions derived for ( 2 ) would also apply to the reaction

$$
\begin{aligned}
& \mu^{-}(k)+N(p) \rightarrow L^{0}\left(k^{\prime}\right)+\text { "anything" } \\
& \longrightarrow \mu^{-}(K)+\mu^{+}(Q)+\nu(\bar{k}) \\
& \mu^{-}(K)+e^{+}(Q)+\nu_{e}(\bar{k})
\end{aligned}
$$

The corresponding expressions for incident $\bar{\nu}$ or $\mu^{+}$producing charged and neutral heavy leptons con be trivially obtained from expressions for reaction ( 2 ).

\section{a). The Production Process:}

The amplitude, ${ }^{A} \lambda \lambda^{\prime}$ ', for the production of a heavy lepton of helicity $\lambda$ ' from an incident neutrino of helicity $\lambda$ can be expressed as 7

$$
A_{\lambda \lambda^{\prime}}=\frac{G_{1}}{\sqrt{2}} U_{L}\left(k^{\prime}, \lambda^{\prime}\right) \gamma\left(1+h \gamma_{5}\right) U_{\nu}(k, \lambda)
$$

where $J$ is the hadronic current, $G$ is the analog of the Fermi constant for the coupling of the heavy lepton to the nucleon at the production vertex (see Fig. I), and $h= \pm l$ if the heavy lepton couples via $(V \mp A)$ respectively. We describe the hadronic vertex of the reaction in terms of the structure functions defined by 4 


$$
\begin{aligned}
W_{\mu \nu}= & -\delta_{\mu \nu} W_{1}-\frac{1}{M^{2}} p_{\mu} p_{\nu} W_{2}-\frac{1}{2 M^{2}} \epsilon_{\mu \nu \alpha \beta} p_{\alpha} q_{\beta} W_{3}=\frac{1}{M^{2}} q_{\mu} q_{\nu} W_{4} \\
& -\frac{1}{2 M^{2}}\left(p_{\mu} q_{\nu}+p_{\nu} q_{\mu}\right) W_{5}
\end{aligned}
$$

where $M$ is the mass of the target nucleon, and $q=k-k^{\prime}$, the momentum: transfer from the leptons to the hadrons. The state of the produced lepton $L$ in its rest frame can then be described by a $(2 \times 2)$ density matrix of the form

$$
\rho_{0}=\frac{1}{2}\left(1+\vec{\sigma} \cdot \frac{\vec{B}_{0}}{A_{0}}\right)
$$

where $\sigma$ 's are $2 \times 2$ Pauli matrices, and the vector $\frac{\vec{B}_{0}}{A_{0}}$ gives the polarization of $L$ in its rest frame. In this frame, where $\vec{k}^{\prime}=\vec{k}-\vec{q}=0, \vec{B}_{0}$ is expressed, for convenience in subsequent generalization to a covariant frame, in terms of the dependent 3-vectors, $\vec{k}, \vec{q}$, and $\vec{p}$ as

$$
\begin{aligned}
\vec{B}_{0}= & -\frac{2 h G_{1}^{2} m_{L}}{8 \pi^{2} k \cdot p}\left\{\vec{k}\left[2 W_{1}+\frac{W_{2}}{M^{2}} p^{2}+\frac{W_{4}}{M^{2}} q^{2}+\frac{W_{5}}{M^{2}} q \cdot p\right]\right. \\
& +\vec{p}\left[-2 \frac{W_{2}}{M^{2}} k \cdot p-\frac{1+h^{2}}{2 h} \frac{W_{3}}{M^{2}} q \cdot k-\frac{W_{5}}{M^{2}} q \cdot k\right] \\
& \left.+\vec{q}\left[\frac{1+h^{2}}{2 h} \frac{W_{3}}{M^{2}}-2 \frac{W_{4}}{M^{2}} k \cdot q-\frac{W_{5}}{M^{2}} k \cdot p\right]\right\}
\end{aligned}
$$

and 


$$
\begin{aligned}
A_{0}= & -\frac{G_{1}^{2}\left(1+h^{2}\right) m_{L}}{8 \pi^{2} k \cdot p} \cdot\left\{2 k_{0} W_{1}+\frac{W_{2}}{M^{2}}\left(k_{0} p^{2}-2 p_{0} k \cdot p\right)-\frac{W_{3}}{M^{2}} \frac{2 h}{1+h^{2}}\left(p_{0} k \cdot q-q_{0} k \cdot p\right)\right. \\
& \left.+\frac{W_{4}}{M^{2}}\left(q^{2} k_{0}-2 q_{0} k \cdot q\right)+\frac{W_{5}}{M^{2}}\left(k_{0} p \cdot q-p_{0} q \cdot k-q_{0} k \cdot p\right)\right\}
\end{aligned}
$$

where $k_{0}, q_{0}$ and $p_{0}$ are the $o^{\text {th }}$ components of $k, q$ and $p$ and $m_{L}$ is the mass of heavy lepton $L$.

The cross section for production of heavy leptons is directly related to the function $A_{0}$ and is given in the lab frame by.

$$
\begin{aligned}
& \frac{d \sigma_{p}}{d q^{2} d \nu}=-\frac{G_{1}^{2}\left(1+h^{2}\right)}{8 M E^{2} \pi}\left\{2 k^{\prime} \cdot k W_{1}+\frac{W_{2}}{M^{2}}\left(k^{\prime} \cdot k p^{2}-2 k^{\prime} \cdot p k \cdot p\right)\right. \\
& \left.+\frac{2 h}{1+h^{2}} \frac{W_{3}}{M^{2}}\left(k^{\prime} \cdot q k \cdot p-k^{\prime} \cdot p k \cdot q\right)+\frac{W_{4}}{M^{2}}\left(k^{\prime} \cdot k q^{2}-2 k^{\prime} \cdot q k \cdot q\right)\right) \\
& \left.+\frac{W_{5}}{M^{2}}\left(k^{\prime} \cdot k p \cdot q-k^{\prime} \cdot p k \cdot q-k^{\prime} \cdot q k \cdot p\right)\right\}
\end{aligned}
$$

where $E$ is the incident lepton energy in the lab frame, and $V=-q \cdot p / M$.

b). Decay of the Heavy Lepton:

The amplitude for the decay of a heavy lepton $L$ of helicity $\lambda^{\prime}$ to a $\nu$ of helicity $\lambda_{f}$ via a reaction of the type

$$
L^{-}\left(k^{\prime}, \lambda^{\prime}\right) \longrightarrow \nu\left(K, \lambda_{f}\right)+\ell^{-}(Q)+\bar{\nu}_{l}(\bar{k})
$$


is expressed as

$$
\begin{aligned}
& { }_{\lambda^{\prime} \lambda_{f}}=\frac{G_{2}}{\sqrt{2}}\left[\bar{U}_{\nu}\left(K, \lambda_{f}\right) \gamma_{\mu}\left(1+g \gamma_{5}\right) U_{L}\left(k^{\prime}, \lambda^{\prime}\right)\right] \\
& \times\left[\begin{array}{lll}
\bar{U}_{l}(Q) & \gamma_{\mu}\left(1+\gamma_{5}\right) & v_{\nu_{l}}(\bar{k})
\end{array}\right]
\end{aligned}
$$

where again $g= \pm 1$ depending on whether $L$ couples via $\left(V_{\mp} A\right)$ respectively.

In the rest frame of the heavy lepton, the decay matrix for the process can be written as

$$
D_{0}=\gamma_{0}\left[1+\vec{\sigma} \cdot \frac{\vec{\beta}_{0}}{\gamma_{0}}\right]
$$

where

$$
\gamma_{0}=-32 G_{2}^{2} m_{L}\left[(1+g)^{2} \bar{k}_{0} K \cdot Q+(1-g)^{2} Q_{0} K \cdot \bar{k}\right]
$$

and

$$
\beta_{0}=-32 G_{2}^{2} m_{L}\left[(1+g)^{2} \overrightarrow{\vec{k}} K \cdot Q+(1-g)^{2} \vec{Q} K \cdot \vec{k}\right]
$$

The rate for the decay of a polarized heavy lepton to leptons is then given by

$$
\Gamma_{L \rightarrow l}=\frac{1}{(2 \pi)^{5}} \frac{1}{2 m_{L}} \int \frac{d^{3} Q}{2 Q_{0}} \frac{d^{3} K}{2 K_{0}} \frac{d^{3} \vec{k}}{2 k_{0}} \delta^{4}\left[k^{1}-K-Q-\bar{k}\right] \frac{1}{2} \operatorname{Tr}\left[\rho D_{0}\right]
$$

where $\rho$ is the density matrix specifying the polarization state of the heavy lepton.

ع). Production and Subsequent Decay of the Heavy Lepton:

Taking the trace of the matrix $\left(\rho_{0} D_{0}\right)$ and generalizing to a covariant form we get the required expression for the cross section for process of Fig. 1 as 


$$
\sigma=\frac{G_{1}^{2} G_{2}^{2}}{8(2 \pi)^{7} \sqrt{(k \cdot p)^{2}-m_{l}^{2} \cdot M^{2} m_{L} \Gamma}} I
$$

where

$$
I=\int \frac{d^{3} k^{\prime}}{2 k_{0}^{\prime}} \frac{d^{3} Q}{2 Q_{0}} \frac{d^{3} \bar{k}}{2 \bar{k}_{0}} \frac{d^{3} K}{2 K_{0}} \quad \delta^{4}\left(k^{\prime}-\bar{k}-K-Q\right) T
$$

$m_{\ell}$ is the mass of the incident lepton, $\Gamma$ the total width ${ }^{9}$ of $L$, and

$$
\begin{aligned}
& T=64(1+g)^{2} K \cdot Q\left\{2 W_{1}\left[2 h m_{L}^{2} k \cdot \bar{k}+(1+h)^{2} k^{1} \cdot k k^{\prime} \cdot \bar{k}\right]\right. \\
& +\frac{W_{2}}{M^{2}}\left[2 h m_{L}^{2}\left(p^{2} k \cdot \bar{k}-2 k \cdot p \bar{k} \cdot p\right)+(1+h)^{2} k^{\prime} \cdot \bar{k}\left(k^{\prime} \cdot k p^{2}-2 k^{\prime} \cdot p k \cdot p\right)\right] \\
& -\frac{W_{3}}{M^{2}}\left[(1+h)^{2} k^{\prime} \cdot \bar{k}\left(k^{\prime} \cdot p k \cdot q-k^{\prime} \cdot q k \cdot p\right)+\left(1+h^{2}\right) m_{L}{ }^{2}(k \cdot q \bar{k} \cdot p-k \cdot p \bar{k} \cdot q)\right] \\
& +\frac{W_{4}}{M^{2}}\left[2 h m_{L}^{2}\left(q^{2} k \cdot \bar{k}-2 k \cdot q \bar{k} \cdot q\right)+(1+h)^{2} k^{\prime} \cdot \bar{k}\left(k^{\prime} \cdot k q^{2}-2 k^{\prime} \cdot q k \cdot q\right)\right] \\
& \left.+\frac{W_{5}}{M^{2}}\left[2 h m_{L}^{2}(q \cdot p k \cdot \bar{k}-q \cdot k p \cdot \bar{k}-q \cdot \bar{k} k \cdot p)+(1+h)^{2} k^{\prime} \cdot \bar{k}\left(k^{\prime} \cdot k p \cdot q-k^{\prime} \cdot p k \cdot q-k^{\prime} \cdot q k \cdot p\right)\right]\right\} \\
& +64(1-g)^{2} K \cdot \bar{k}\left\{2 W_{1} \quad\left[-2 h m_{L}^{2} k \cdot Q+(1-h)^{2} k^{\prime} \cdot k k^{\prime} \cdot Q\right]\right. \\
& +\frac{W_{2}}{M^{2}}\left[-2 h m_{L}^{2}\left(p^{2} k \cdot Q-2 k \cdot p p \cdot Q\right)+(1-h)^{2} k^{\prime} \cdot Q\left(k^{\prime} \cdot k p^{2}-2 k^{\prime} \cdot p k \cdot p\right)\right] \\
& -\frac{W_{3}}{M^{2}}\left[(1-h)^{2} k^{\prime} \cdot Q\left(k^{\prime} \cdot q k \cdot p-k^{\prime} \cdot p k \cdot q\right)+\left(1+h^{2}\right) m_{L}^{2}(k \cdot p q \cdot Q-k \cdot q p \cdot Q)\right] \\
& +\frac{W_{4}}{M^{2}}\left[-2 h m_{L}^{2}\left(q^{2} k \cdot Q-2 k \cdot q q \cdot Q\right)+(l-h)^{2} k^{\prime} \cdot Q\left(q^{2} k^{\prime} \cdot k-2 k^{\prime} \cdot q k \cdot q\right)\right] \\
& \left.+\frac{W_{5}}{M^{2}}\left[-2 h m_{L}{ }^{2}(q \cdot p Q \cdot k-k \cdot p q \cdot Q-k \cdot q p \cdot Q)+(1-h)^{2} k^{\prime} \cdot Q\left(k^{\prime} \cdot k q \cdot p-k^{\prime} \cdot p k \cdot q-k^{\prime} \cdot q k \cdot p\right)\right]\right\}
\end{aligned}
$$


It may be worth mentioning that expressions (16) to (18), for the cross section for production and decay of charged heavy leptons, $L^{ \pm}$, via reactions of the type (2) are exact. However, for the production and decay of neutral heavy leptons, $L^{\circ}$ or $\overline{L^{0}}$, via reactions of types $(3)$ and $(4)$, the expressions for the cross section obtained above is correct only to the extent that the incident lepton mass $m_{l}=0$.

d). Phase Space Integration and the Differential Cross Section:

Neglecting the electron and muon mass, the phase space integration, over the unobserved pair of leptons in the decay products, is carried out analytically yielding $I$ of the general form:

$$
I=\int \frac{d^{3} k^{\prime}}{2 k_{0}^{\prime}} \frac{d^{3} Q}{2 Q_{0}} \quad F\left(k^{\prime} \cdot Q\right) \theta\left[-\left(k^{\prime}-Q\right)^{2}\right]
$$

where $F$ is a quadratic function of $\left(k^{\prime} \cdot Q\right)$ and is given in the appendix. We next introduce a coordinate system in the lab frame, shown in Fig. 2, in which the incident lepton beam with 3 -momenta $\vec{k}$ is along the $Z$ axis, $\vec{k}$ ' the 3-momentum of the produced heavy lepton lies in the $Z X$ plane, making an angl:s $\theta^{\prime}$ with the $Z$ axis and $\theta$ and $\varnothing$ are the polar and azimuthal angles of $\vec{Q}$, the 3-momentum of the charged lepton which is under observation. Since $I$, in equation (19) is quadratic in $\cos \varnothing$, the integration over $\varnothing$ can be done trivially to yield:

$$
\begin{aligned}
\frac{d \sigma}{d q^{2} d \nu d Q_{t}^{2} d Q_{\ell}} & =\frac{G_{1}^{2} G_{2}^{2}}{48(2 \pi)^{4} m_{L} \Gamma M E^{2} Q_{0}}\left(A_{O Q}+\frac{A_{2 Q}}{2}\right) \Phi_{Q} \\
& \left.+A_{1 Q} \sin \Phi_{Q}+\frac{A_{2 Q}}{4} \sin \left(2 \Phi_{Q}\right)\right]
\end{aligned}
$$


where $Q_{\ell}, Q_{t}$ are the longitudinal and transverse components of $\vec{Q}, A_{1 Q}, A_{2 Q}$ are the coefficients of $\cos \varnothing$ and $(\cos \varnothing)^{2}$ and $A_{O Q}$ is the term in (19) that is independent of $\varnothing$. These are given in the appendix and $\Phi_{Q}$, the upper limit on $\varnothing$, is given by ${ }^{10}$

$$
\Phi_{Q}^{\prime}=\cos ^{-1}\left(\frac{2 k_{0}^{\prime} Q_{0}-2 k_{\ell}^{\prime} Q_{\ell}-m_{L}^{2}}{2 k_{t}^{\prime} Q_{t}}\right)
$$

For the decay of a ne utral heavy lepton into two charged leptons and a neutrino, as in (3) and (4), one may also be interested in the differential cross section of the other charged particle of 3-momentum $\vec{K}$. This is the same in form as (20), that is,

$$
\begin{aligned}
\frac{d \sigma}{d q^{2} d \nu d K_{t}^{2} d K_{l}} & =\frac{G_{1}^{2} G_{2}^{2}}{48(2 \pi)^{5} m_{L} \Gamma_{E}^{2} M K_{0}}\left[\left(A_{O K}+\frac{A_{2 K}}{2}\right) \Phi_{K}\right. \\
& \left.+A_{K K} \sin \Phi_{K}+\frac{A_{2 K}}{4} \sin \left(2 \Phi_{K}\right)\right]
\end{aligned}
$$

where again,

$$
\Phi_{K}=\cos ^{-1}\left(\frac{2 k_{0}^{\prime} K_{0}-2 k_{\ell}^{\prime} k_{l}-m_{L}^{2}}{2 k_{t}^{\prime} K_{t}}\right)
$$

The coefficients $A_{O K}, A_{1 K}$ and $A_{2 K}$ are again given in the appendix.

e). Differential Cross Section Neglecting Polarization of $L$ :

The cross section, $d \sigma_{0}$, neglecting polarization of the heavy lepton, is obtained by taking the product of $A_{0} \gamma_{0}$ instead of $\rho_{0} D_{0}$ from equations $(\lambda)$ and (12) and is given by 
10.

$$
\begin{aligned}
\frac{d \sigma_{0}}{d q^{2} d \nu d Q^{2} d Q_{l}} & =\frac{G_{1}^{2} G_{2}^{2}}{48(2 \pi)^{5} m_{L} r M E^{2} Q_{0}}\left[\left(A_{00}+\frac{A_{02}}{2}\right) \Phi_{Q}\right. \\
& \left.+A_{01} \sin \Phi_{Q}+\frac{A_{02}}{4} \sin \left(2 \Phi_{Q}\right)\right]
\end{aligned}
$$

where $A_{00}, A_{01}$, and $A_{02}$ are again given in the appendix.

f). Differential Cross Section along the Beam Axis:

Along the beam axis $Q_{\mathrm{f}},\left(\right.$ or $\left.K_{\mathrm{f}}\right)=0$ and $\Phi_{Q},\left(\right.$ or $\left.\Phi_{K}\right)=\pi$; and since $Q_{0}=\left|Q_{\ell}\right|, K_{0}=\left|K_{\ell}\right|$, the differential cross section (20) and (22) becomes linear functions of $Q_{\ell}$ (or $K_{\ell}$ ) which can be analytically integrated to yield,

$$
\left.\frac{d \sigma}{d q^{2} d \nu d Q_{t}^{2}}\right|_{Q_{t}=0}=c_{-}(1-g)^{2}+c_{+}(1+g)^{2}
$$

where $C_{-}$and $C_{+}$are given in the appendix. On the other hand

$$
\left.\frac{d \sigma}{d q^{2} d \nu d K_{t}^{2}}\right|_{K_{t}=0}=\frac{G_{2}^{2}\left(1+g^{2}\right) m_{L}^{3}}{48 \pi^{3} r} \frac{d \sigma_{p}}{d q^{2} d \nu}
$$

So that for both $V+A$ and $V-A$ couplings

$$
\left.\frac{d \sigma}{d K_{t}^{2}}\right|_{K_{t}=0}=\frac{G_{2}^{2} m_{L}^{3}}{24 \pi^{3}} \frac{\sigma_{p}}{\Gamma} .
$$

or using ( 15$)$ we get 11

$$
\left.\frac{d r}{d \bar{K}_{t}^{2}}\right|_{K_{f}=0}=\frac{8}{m_{L}^{2}} \sigma_{p} R
$$


11.

where $R$ is the branching ratio defined as

$$
R=\frac{r_{L \rightarrow \ell}}{r}
$$

From ( 24 ) and the appendix we also notice that only for $V-A$, ie. for $g=1$,

$$
\left.\frac{d \sigma}{d Q_{t}^{2}}\right|_{Q_{t}=0} \underset{g \rightarrow 1}{\longrightarrow} \frac{8}{m_{L}^{2}} \sigma_{P} R .
$$

Finally, from ( 24 ) we get

$$
\left.\frac{d \sigma_{0}}{d Q_{t}^{2}}\right|_{Q_{t}=0}=\frac{\left[5\left(1+g^{2}\right)-2 g\right]}{m_{L}^{2}} \sigma_{p} R
$$

So that for $V-A$

$$
\left.\frac{d \sigma_{0}}{d Q_{t}^{2}}\right|_{Q_{t}=0} \underset{g \rightarrow 1}{\longrightarrow} \frac{8}{m_{L}^{2}} \sigma_{p} R
$$

and for $V+A$

$$
\left.\frac{d \sigma_{0}}{d Q_{t}^{2}}\right|_{Q_{t}=0} \underset{g \rightarrow-1}{\longrightarrow} \frac{12}{m_{L}^{2}} \sigma_{p} R
$$


III. Numerical Computations

Numerical computations are done for $E=50,100,200$ and $500 \mathrm{GeV}$. The couplings $G_{1}$ and $G_{2}$ are taken identical to that for the muon. The branching ratio, $R$, into any one of the leptonic decay modes of $L$ is taken to be ${ }^{12} 15 \%$; Introducing $\omega^{\prime}=\left(2 M \nu+M^{2}\right) / q^{2}$ the structure functions are parameterized as follows 4,5 :

$$
\begin{aligned}
& F_{2}\left(\omega^{\prime}\right)=1 \cdot 1\left(1-\left(\frac{1}{\omega^{3}}\right)^{2}\right)^{3} \\
& F_{1}\left(\omega^{\prime}\right)=\frac{\omega^{\prime}}{2} F_{2}\left(\omega^{\prime}\right) \\
& F_{3}\left(\omega^{\prime}\right)=-|B| \omega^{\prime} F_{2}\left(\omega^{\prime}\right) \\
& F_{4}\left(\omega^{\prime}\right)=0 \\
& F_{5}\left(\omega^{\prime}\right)=2 F_{1}\left(\omega^{\prime}\right)
\end{aligned}
$$

where $F_{1}=W_{1}$ and $F_{i}=\frac{\nu}{M} W_{i}$ with $i=2,3,4$ and 5 . As indicated by the CERN Gargamelle data on $\bar{v}, \nu$ cross section ratio we take $B=1 .^{5}$ The total cross section $\sigma_{p}$ as a function of the heovy lepton mass obtained by this parameterization, is shown in Fig. 3, for $E=50,100,200$ and $500 \mathrm{GeV}_{3}$. We find that $\sigma_{p}$ for $: m_{L}=m_{\mu}$ in Fig. 3 compares very well with the experimental result.

$$
\sigma_{p}^{V}=(0.8 \pm 0.2) \times 10^{-38} \mathrm{E} \mathrm{cm}^{2}
$$


Furttier, our parameterization ( 34 ) with $|B|=1$ also yields $\frac{\sigma_{p}(\bar{\nu})}{\sigma_{p}(\nu)}=\frac{1}{3}$ in agreement with the observed values. 5

To obtain $\frac{d \sigma}{d Q_{t}^{2}}, \frac{d \sigma}{d K_{t}^{2}}, \frac{d \sigma_{0}}{d Q_{t}^{2}}$, three dimensional numerical integration of (20), (22) and ( 24$)$ is done using the IBM systems 360 , Scientific Subroutine Package, RANDU, for generating random numbers. $3 \times 10^{4}$ points are taken for each value of. $Q_{f}$ or $K_{f}$. Statistical fluctuation in the differential cross sections are estimated to be $\leqslant 2 \%$. 


\section{Discussion of Results}

To get a feeling for the cross section for production of heavy leptons we draw a graph for $\sigma_{p}$ versus $m_{L}$ (Fig. 3) for incident lepton energies of $E=50$, 100,200 and $500 \mathrm{GeV}$ in the lab frame. They exhibit a fast drop in the cross section with an increase in $\mathrm{m}_{\mathrm{L}}$. For example, for $E=100 \mathrm{GeV}$, when the kinematical limit is $m_{L} \leqslant 13 \mathrm{GeV}$, the cross section drops from $5.7 \times 10^{-37} \mathrm{~cm}^{2}$ for $m_{L}=2 \mathrm{GeV}$ to $3.0 \times 10^{-41} \mathrm{~cm}^{2}$ for $m_{L}=12 \mathrm{GeV}$, a drop of $\sim 4$ orders of magnitude over a $10 \mathrm{GeV}$ increase in mass.

The differential cross section distributuion with respect to (transverse momentum $)^{2}$, assuming $(V-A)$ coupling for $L$, is shown in grophs $4,5,6$ and 7 . In figures 4 and 5 the incident particle is $\nu$ or a left handed $\mu^{-}$and in figures 6 and 7 the incident particle is $\vec{\nu}$ or a right handed $\mu^{+}$. These curves exhibit a very sharp drop with increase in transverse momentum. In fact, a numerical estimate of $\left\langle Q_{t}^{2}\right\rangle$ shows that for $E=100 \mathrm{GeV}$ and $m_{L}=5 \mathrm{GeV},\left\langle Q_{t}^{2}\right\rangle=3.8 \mathrm{GeV}^{2}$ when the kinematical limit is $Q_{t}^{2} \leq 45 \mathrm{GeV}^{2}$. The cross section $\frac{d \sigma}{d Q_{t}{ }^{2}}$ again

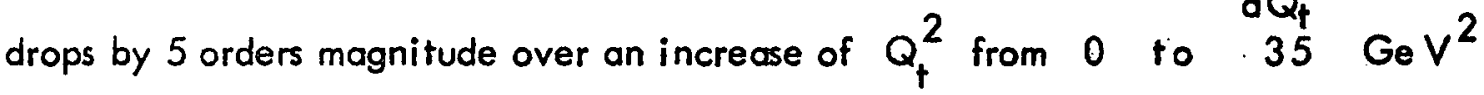
for $E=100 \mathrm{GeV}$. For $(V-A)$ coupling of $L, \frac{d \sigma}{d Q_{t}^{2}}$ and $\frac{d \sigma}{d K_{t}^{2}}$ are identical as exhibited by $(18)^{8}$. Again, it may be worth reminding that the branching ratio, $R$, throughout these computations has been taken to be $15 \%$, and, in adapting these graphs to various models for heavy leptons, one must normalize them to the appropriate value of $R$ given by the individual models. ${ }^{12}$

In Figs. 8 and 9 we redraw Figs. 4 and 5 by normalizing $\frac{d \sigma}{d Q_{t}^{2}}$ to $\frac{d \sigma}{d Q_{t}^{2}}$ 


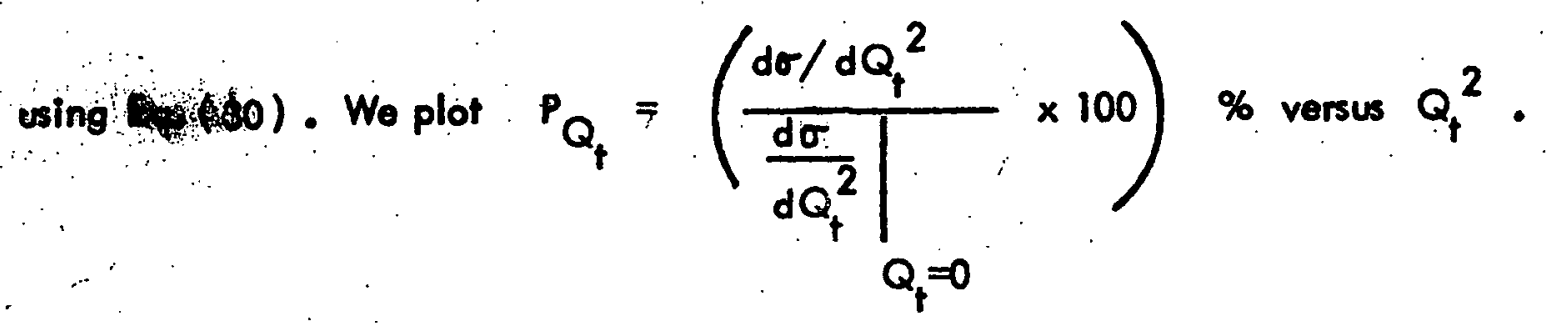

These graphs are therefore independent of the branching ratio and hence can be : directly compared to an experimental curve of $\left(\frac{\text { number of events with } Q_{t}^{2}}{\text { number of events with } Q_{t}=0}\right)$ versus $Q_{f}^{2}$. In Fig. 8 we keep the energy fixed at $E=100 \mathrm{GeV}$ and vary the moss $m_{L}$ from 2 to $11 \mathrm{GeV}$, and, in Fig. 9 we keep the mass fixed at $5 \mathrm{GeV}$ and vary the energy from 50 to $500 \mathrm{G}$ V. Fig. 8 exhibits a very strong dependence of $P_{Q_{f}}$ on $m_{L}$ wheroes Fig. 9 shows practically no dopendence of $P_{Q_{f}}$ on $E$, for the kinematically allowed values of $Q_{t}^{2}$ for each $E$.

If the $(V+A)$ coupling is instead assumed for $L$ then the differential cross sections for incident $\nu$ or right handed $\mu^{-}$is shown in Figures 10 and 11 , and, for incident $\bar{v}$ or left handed $\mu^{+}$is shown in Figs. 12 and 13. For $(V+A)$ coupling $\frac{d \sigma}{d Q_{t}{ }^{2}} \neq \frac{d \sigma}{d K_{t}^{2}}$ and we therefore draw both the distributions. In our calculation we have assumed $L^{-}$to have the same lepton number as $\ell^{-}$, and $\nu_{l}$. If instiead $L^{+}$is taken to have the same lepton number os $\ell^{-}$and $\nu_{l^{\prime}}$ as is done in a number of gauge theories, then our graphs for the differential cross sections of the emerging charged leptons will get interchanged for the $(V+A)$ and $(V-A)$ coses.

The effects due to polarization of the heavy lepton are shown in Figures 14 and 15. The density matrix of the heavy lepton; expressed in Equations 7,8 and 9, is very convenient for this purpose. The polarization vector $\hat{s}$ in the rest frame of Li given by 


$$
\hat{S}=\frac{\overrightarrow{B_{0}}}{A_{0}}
$$

where $B_{0}, A_{0}$ are given in Equations ( 8 ) and $(9)$, is used to obtain the degree of left handedness, $P_{L H}$, of $L$ defined as:

$$
P_{L H}=\frac{1-\hat{S} \cdot \hat{k}^{\prime}}{2} \times 100 \%
$$

Fig. 14 shows $P_{L H}$ as a function of the heavy lepton energy, $k^{\prime}{ }_{0}^{\prime}$, keeping the scattering angle $\theta$ fixed in the lab frame. The figure shows effects of changing the scattering angle $\theta^{\prime}$, which, incidentially, has to be very small because of the constraint $q^{2} \leq 2 M \nu$, the incident lepton energy $E$ and the mass $m_{L}$ of the heavy lepton.

The consequence of all these dependences of $\hat{S}$ on $k^{\prime}{ }_{0}^{\prime}, \dot{\theta}$ and $E$ on the distributions of the emerging charged lepton is shown in Fig. 15. For this purpose we plot two functions $D_{0}$ and $D_{L}$ where

$$
D_{0}=\frac{d \sigma / d Q_{t}^{2}-d \sigma_{0} / d Q_{t}^{2}}{d \sigma / d Q_{t}^{2}}
$$

and

$$
D_{L}=\frac{d \sigma / d Q_{t}^{2}-d \sigma_{L} / d Q_{t}^{2}}{d \sigma / d Q_{t}^{2}}
$$

Here $\frac{d \sigma}{d Q_{t}^{2}}$ is the distribution which incorporates all the polarization effects of the heavy lepton, $\frac{d \sigma_{0}}{d Q_{t}^{2}}$ is obtained by assuming the heavy lepton to be completely unpolairzed and $\frac{d \sigma_{L}}{d Q_{t}^{2}}$ assuming the heavy lepton to be completely left handed. In the region of experimental interest, $Q_{t} \geqslant 1.5 \mathrm{GeV}$, one 
findstat the polarization effects are rather significant.

\section{Acknowledgement}

I am deeply indebted to Professor Norman. Christ for this sponsorship of this problem and for constant encouragement and advice in the course of this work. 
18.

Appendix

a). Coefficients $A_{O Q}, A_{1 Q}$, and $A_{2 Q}$ of equation (20):

From (19):

$$
I=\int \frac{d^{3} k^{\prime}}{k_{0}^{\prime}} \frac{d^{3} Q}{Q_{0}} F\left(k^{\prime} \cdot Q\right) \theta\left[-\left(k^{\prime}-Q\right)^{2}\right]
$$

where $F\left(k^{\prime} \cdot Q\right)$ is a quadratic function of $\left(k^{\prime} \cdot Q\right)$. Hence we may rewrite this as a quadratic function of $\cos \emptyset$. We write, for convenience,

$$
F\left(k^{\prime} \cdot Q\right)=B_{Q}-m_{L}^{2} A_{Q}
$$

where

$$
B_{Q}=B_{0}+B_{1} \cos \phi+B_{2}(\cos \varnothing)^{2}
$$

and

$$
A_{Q}=A_{0}+A_{i} \cos \varnothing
$$

with

$$
B_{0}=\left(k_{l}^{\prime} Q_{l}-k_{0}^{\prime} Q_{0}\right)\left[C_{0}+C_{k} k\left(Q_{l}-Q_{0}\right)-C_{p} M Q_{0}+C_{k^{\prime}}\left(k_{l}^{\prime} Q_{l}-k^{\prime} Q_{0}\right)\right]
$$

$$
B_{1}=k_{t}^{\prime} Q_{t}\left[C_{0}+C_{k} k\left(Q_{l}-Q_{0}\right)-C_{p} M Q_{0}+C_{k^{\prime}}\left(k_{l}^{\prime} Q_{l}-k_{0}^{\prime} Q_{0}\right)\right]
$$

$$
B_{2}=C_{k^{\prime}} k_{t}^{2} Q_{t}^{2}
$$

$$
A_{0}=k A_{k}\left(Q_{l}-Q_{0}\right)-A_{p} M Q_{0}+A_{k^{\prime}}\left(k_{l}^{\prime} Q_{l}-k_{0}^{\prime} Q_{0}\right)
$$

and

$$
A_{1}=k_{t}^{\prime} Q_{t} A_{k^{\prime}}
$$


19.

The subscripts $k, p, k^{\prime}$ in the coefficients $C^{\prime}$ 's and $A^{\prime} s$ imply that the latter are coefficients of $k \cdot Q, p \cdot Q$, and $k^{\prime} \cdot Q$ respectively and subscript 0 denotes the term independent of $Q$ in $F\left(k^{\prime} Q\right)$.

The coefficients that appear in equation (20) are then given as

$$
\begin{aligned}
& A_{0, Q}=B_{0}-m_{L}^{2} A_{0} \\
& A_{1 Q}=B_{1}-m_{L}^{2} A_{1} \\
& A_{2 Q}=B_{2} .
\end{aligned}
$$

So we need to know $C_{0}, C_{k}, C_{p}, C_{k^{\prime}}$ and $A_{k}, A_{p}, A_{k^{\prime}}$ of equations (A.5) to (A.9) above. These are given below.

$$
C_{0}=-2(1+g)^{2}\left(1+h^{2}\right) m_{L}^{2} C_{o t}
$$

where

$$
\begin{aligned}
C_{\text {of }}= & 2 W_{1} k^{\prime} \cdot k+\frac{W_{2}}{M^{2}}\left(k^{\prime} \cdot k p^{2}-2 k^{\prime} \cdot p k \cdot p\right)-\frac{W_{3}}{M^{2}}\left(k^{\prime} \cdot p k \cdot q-k^{\prime} \cdot q k \cdot p\right) \frac{2 h}{1+h^{2}} \\
& +\frac{W_{4}}{M^{2}}\left(k^{\prime} \cdot k q^{2}-2 k^{\prime} \cdot q k \cdot q\right)+\frac{W_{5}}{M^{2}}\left[\left(k^{\prime} \cdot k \cdot \cdot q-k^{\prime} \cdot p k \cdot q-k^{\prime} \cdot q k \cdot p\right)\right] \\
C_{p}= & 8 h m_{L}^{2}\left[(1+q)^{2}-3(1-q)^{2}\right] C_{p:}
\end{aligned}
$$

where

$$
\begin{aligned}
& C_{p t:}=2 \frac{W_{2}}{M^{2}} k \cdot p+\frac{W_{3}}{M^{2}} \frac{1+h^{2}}{2 h} k \cdot q+\frac{W_{5}}{M^{2}} k \cdot q \\
& C_{k}=-8 m_{L}^{2}\left[(1+g)^{2}-3(1-g)^{2}\right] C_{k t}
\end{aligned}
$$

where

$$
C_{k+}=2 W_{1}+\frac{W_{2}}{M^{2}} p^{2}+\frac{W_{3}}{M^{2}} \frac{1+h^{2}}{2 h} k \cdot p+\frac{W_{4}}{M^{2}}\left(q^{2}-2 q \cdot k\right)+\frac{W_{5}}{M^{2}}(q \cdot p-k \cdot p)
$$


20.

$$
\begin{aligned}
C_{k^{\prime}}= & -4\left[3(1-g)^{2}(1-h)^{2}+(1+g)^{2}(1+h)^{2}\right] C_{k^{\prime} t} \\
& -4\left[(1+g)^{2}(1+h)^{2}-3(1-g)^{2}(1-h)^{2}\right] C_{k^{\prime} t_{2}} \\
& +8 h m_{L}^{2}\left[3(1-g)^{2}-(1+g)^{2}\right] C_{k^{\prime} t_{3}}
\end{aligned}
$$

where

$$
\begin{aligned}
& C_{k^{\prime}+1}=C_{\text {ot }}+\frac{W_{3}}{M^{2}}\left(k^{\prime} \cdot p k \cdot q-k^{\prime} \cdot q k \cdot p\right) \frac{2 h}{1+h^{2}} \\
& C_{k^{\prime}+2}=\frac{W_{3}}{M^{2}}\left(k^{\prime} \cdot q k \cdot p-k^{\prime} \cdot p k \cdot q\right) \\
& C_{k^{\prime}+3}=2 \frac{W_{4}}{M^{2}} k \cdot q+\frac{W_{5}}{M^{2}} k \cdot p-\frac{W_{3}}{M^{2}} \frac{1+h^{2}}{2 h} k \cdot p
\end{aligned}
$$

Next,

$$
\begin{aligned}
& A_{k}=2 h m_{L}^{2}\left[(1+g)^{2}-6(1-g)^{2}\right] C_{k t} \\
& A_{p}=-2 h m_{L}^{2}\left[(1+g)^{2}-6(1-g)^{2}\right] \cdot C_{p t}
\end{aligned}
$$

and

$$
\begin{aligned}
A_{k^{\prime}}= & {\left[6(1-g)^{2}(1-h)^{2}+(1+g)^{2}(1+h)^{2}\right] C_{k^{\prime}+1} } \\
& +\left[(1+h)^{2}(1+g)^{2}-6(1-g)^{2}(1-h)^{2}\right] C_{k^{\prime} t_{2}} \\
& +2 h m_{L}^{2}\left[(1+g)^{2}-6(1-g)^{2}\right] C_{k^{\prime}+3}
\end{aligned}
$$


21.

b). Coefficients $A_{O K}, A_{1 K}$ and $A_{2 K}$ of equation ( 22 ):

These are obtained in the same general way outlined in the last section.

$$
\begin{aligned}
A_{O K K} & =\left(k_{l}^{\prime} K_{l}-k_{0}^{\prime} K_{0}\right) C_{O K}-\left(k_{l}^{\prime} K_{l}-k_{0}^{\prime} K_{0}\right) B_{k^{\prime}}\left[2\left(k_{l}^{\prime} K_{l}-k_{0}^{\prime} K_{0}\right)+m_{L}^{2}\right] \\
& +B_{p} M K_{0} \cdot\left[2\left(k_{l}^{\prime} K_{l}-k_{0}^{\prime} K_{0}\right)+m_{L}^{2}\right] \\
& -E K_{0}\left(\cos \theta^{\prime}-1\right) B_{k}\left[2\left(k_{l}^{\prime} K_{l}-k_{0}^{\prime} K_{0}\right)+m_{l}^{2}\right]
\end{aligned}
$$

$$
\begin{aligned}
A_{1 K}= & 2 k_{t}^{\prime} K_{t}\left[C_{0 K}-4 B_{k^{\prime}}\left(k_{l}^{\prime} K_{l}-k_{0}^{\prime} K_{o}\right)+2 M K_{0} B_{p}-2 B_{k} E K_{0}\left(\cos \theta^{\prime}-1\right)\right] \\
& -m_{L}^{2} B_{k^{\prime}} k^{\prime}{ }_{t} K_{t} \\
A_{2 K}= & -4 k_{t}^{\prime 2} K_{t}^{2} B_{k^{\prime}}
\end{aligned}
$$

where $C_{O K}, B_{k^{\prime}} B_{p^{\prime}}$ and $B_{k^{\prime}}$ are given below.

$$
C_{O K}=-\left(1+h^{2}\right) m_{L}^{2}\left[(1+g)^{2}+(1-g)^{2}\right] C_{\text {ot }}
$$

and $C_{\text {of }}$ is given in equation $(A, 12)$.

$$
\begin{aligned}
B_{k}= & 2 h\left[(1+g)^{2}-(1-g)^{2}\right] m_{L}^{2} C_{k t} \\
B_{p}= & -2 h\left[(1+g)^{2}-(1-g)^{2}\right] m_{L}^{2} C_{p t} \\
B_{k^{\prime}}= & -\left[(1+g)^{2}(1+h)^{2}+(1-g)^{2}(1-h)^{2}\right] C_{k^{\prime} t_{1}} \\
& +\left[(1-g)^{2}(1-h)^{2}-(1+g)^{2}(1+h)^{2}\right] C_{k^{\prime} t_{2}} \\
& +2 h m_{L}^{2}\left[(1-g)^{2}-(1+g)^{2}\right] \dot{C}_{k^{\prime} t_{3}}
\end{aligned}
$$


22.

where $C_{k t}, C_{p t}, C_{k^{\prime}+}$, and $C_{k^{\prime}+3}$ are given in part a) of this section.

c). $A_{b_{0}}, A_{01}$ and $A_{02}$ of equation (24):

These are again obtained the same way as those in section a).

$$
\begin{aligned}
A_{00}= & -\left(1+h^{2}\right) C_{\text {ot }}\left(k_{l}^{\prime} Q_{l}-k^{\prime}{ }_{0} Q_{0}\right)\left\{9\left(1+g^{2}-\frac{2}{3} g\right) m_{L}^{2}\right. \\
& \left.+16\left(1+g^{2}-g\right)\left(k_{l}^{\prime} Q_{l}-k^{\prime} Q_{0}\right)\right\} \\
A_{01}= & -\left(1+h^{2}\right) C_{0 t} k^{\prime}{ }_{t} Q_{t}\left\{9\left(1+g^{2}-\frac{2}{3} g\right) m_{L}^{2}+32\left(1+g^{2}-g\right)\left(k_{l}^{\prime} Q_{l}-k_{0}^{\prime} Q_{0}\right)\right\} \\
A_{02}= & -16\left(1+h^{2}\right) C_{\text {ot }} k_{t}^{\prime}{ }_{t} Q_{t}^{2}\left(1+g^{2}-g\right)
\end{aligned}
$$

where $C_{\text {of }}$ is given in $(A .12)$.

d). $C_{+}, C_{-}$occuring in equation ( 25$)$ :

$$
C_{+}=+2 C\left(1+h^{2}\right) m_{L}^{4} C_{\text {ot }}
$$

where

$$
c=\frac{G_{1}^{2} G_{2}^{2} \pi}{48(2 \pi)^{5} m_{L} r M E^{2}}
$$

and $C_{\text {of }}$ is given in (A.12).

$$
\begin{aligned}
C_{-}=C_{L} m_{L}^{4}\{ & -\frac{6 k m_{L}^{2} h}{k_{0}^{1}+k_{L}^{\prime}} C_{k t}+\frac{6 h k_{0}^{\prime} m_{L}^{2}}{k_{t}^{\prime 2}+m_{L}^{2}} C_{p t} \\
& \left.+3(1-h)^{2}\left(C_{k^{\prime}+}-C_{k^{\prime}+t_{2}}\right)-6 m_{L}^{2} h C_{k^{\prime}+}\right\}
\end{aligned}
$$

where $C_{k t}, C_{p t}, C_{k^{\prime} t_{1}}, C_{k^{\prime} t_{2}}$ and $C_{k^{\prime} t_{3}}$ are given in equations (A.16), (A.14) (A.18), $(A .19)$ and $(A .20)$. 
1. Some of the more prominent papers on this topic are H. Georgi, and S.L. Glashow, Phys. Rev. Letters 28 , 1494 (1972); B.W. Lee, Phys. Rev. D6, 1.188 (1972); JoPrentki and B. Zumino, Nucl. Phys。 B47, 99 (1972)。

For a more complete list of references and a brief description of such theories see, J.D. Biorken and C.H. Llewellyn Smith, Phys. Rev. D7, 887 (1973).

2. R. Cester, et al., a proposal to NAL on "Feasible Search for Heavy Neutral Muon Predicted by Gauge Theories", Princeton University (unpublished 1973). W. Lee, ef al., "Proposal to Search for Heavy Leptons and Intermediate Boson from Photon-Nucleon and Photon-Nuclei Collisions", NAL Proposal No. 87 (1970). M. Bernadini, et al., paper submitted to the 6th International Symposium on Electron and Photon Interaction at High Energy, Bonn, Germany, August 1973. B. C. Barish, et al., preprint, "Mass Limit on a Positively Charged Heavy Muon", Calif. Inst. of Tech. (unpüblished 1973)。

3. J.D. Bjorken and C.H. Llewellyn Smith, Phys. Rev. D7, 887 (1973).

4. C. H. Albright, Phys, Rev. Letfers 28, 1150 (1972).

5. C. Baltay, invited paper at Los Angeles Meeting of the American Physical Society, December 27, 1972.

6. We are assuming $L^{-}$to have the same lepton number as $L^{-}$or $\nu_{\ell}$. If instead $\mathrm{L}^{+}$. has the same lepton number as $\ell^{-}$or $\nu_{l}$, as happens in certain gauge theories, our graphs, for $(V-A)$ and $(V+A)$ couplings, for the differential cross section of the outgoing charged lepton will just get interchanged. 
7. We use $p \cdot q=\vec{p} \cdot \vec{q}-p_{0} q_{0}$ and in our notation $\gamma_{5}=\left(\begin{array}{cc}0 & -I \\ -I & 0\end{array}\right)$.

8. Note that we are assuming $(V-A)$ coupling for the $\left(e, \nu_{e}\right)$ or $\left(\mu, \nu_{\mu}\right)$ currents even though $L$ may couple either via $(V-A)$ or $(V+A)$ currents.

9. We use the narrow width approximation, that is, $\left|\frac{1}{k^{\prime 2}+m_{L}^{2}-i m_{L} \Gamma}\right|^{2}=\frac{\pi}{m_{L} \Gamma} \delta\left(k^{\prime 2}+m_{L}{ }^{2}\right)$

10. $\Phi_{Q}$ is the upper limit on $\varnothing$. This results from the constraint $\left(k^{\prime}-Q\right)^{2} \leq 0$. 11. From (15),

$$
\Gamma_{L \rightarrow 2}=\frac{G_{2}^{2} m_{L}^{5}}{4 \times 192 \pi^{3}} \quad\left[(1+g)^{2}+(1-g)^{2}\right]
$$

12. See Ref. 3. Assuming $\mathrm{L}^{+}$to have the same lepton number as $\nu_{\ell}$ and $\ell^{-}$ they estimate $\quad R\left(L_{e}^{+} \rightarrow \mu^{+} \nu_{\mu} \nu_{e}\right) \simeq 15 \%$ and

$$
\left.\mathrm{R}_{\mathrm{e}}^{+} \rightarrow \mathrm{e}^{+} \nu_{\mathrm{e}} \nu_{\mathrm{e}}\right) \simeq 30 \% \text {, }
$$

the difference coming from the identity of the two neutrinos in the final state of the second reaction. Throughout our numerical computations we are using $R=15 \%$ 


\section{FIGURE CAPTIONS}

Figure 1: Feyrman diagram for the proces: $v N \rightarrow L^{-}+$anythisig. The inter$\left\lfloor v+l^{-}+\bar{\nu}_{\ell}\right.$

mediate line is faken to be the mass shell. $k$ and $p$ are momenta of the incident $\nu$ and $N$ respectively, $k^{i}$ is the momentum of the heavy lepton $L^{-}$, $K$ is the momentum of the final $\nu$ which is associated with the leptonic current for $L$, and $Q$ and $\bar{k}$ are the momenta of $\mathcal{L}^{-}$and $\bar{\psi}_{\mathcal{B}}$ respectively.

Figure 2: The co-ordinate system used in the lab frame. The incident lepton with 3-momentum $\vec{k}$ is along the $Z$-axis, the heary lepton with momentum $\vec{k}$ makes an angle $\theta^{\prime}$ in the $Z$-axis and lies in the $Z \times$ plane. The outgoing charged lepton with momentum $\vec{Q}$ is defined by the polar angles $\theta$ and the azimuthal angle $\varnothing$.

Figure 3: The production cross section $\sigma_{\mathrm{p}}$ versus heavy lepton mass $\mathrm{m}_{\mathrm{L}}$ for:incident energy $E=50,100,200$ and $500 \mathrm{GeV}$ in the lab frame and assuming $(V-A)$ coupling for $L$. Incident particle is $\nu$ or left handed $\ell^{-}$.

Figures 4 and 5: In these graphs $L$ is assumed to couple through $(V-A)$ interaction. These curves apply to reactions of the type:

$$
\begin{aligned}
& \nu(k)+N\left(p^{\prime}\right) \longrightarrow L^{-}\left(k^{\prime}\right)+\text { "onything" } \\
& \longrightarrow \nu(K)+\ell^{-}(Q)+\bar{\nu}_{\ell}(\bar{k})
\end{aligned}
$$

and

$$
\begin{aligned}
& \ell^{-} H^{(k)}+N(p) \longrightarrow L^{\circ}\left(k^{\prime}\right)+\text { "anything" } \\
& L \ell^{-}(K)+\ell^{+}(Q)+\nu_{\ell^{\prime}}
\end{aligned}
$$

where $\ell_{L H}$ is the left handed electron or muon. For $(V-A)$ coupling of $L$, $\frac{d \sigma}{d Q_{t}{ }^{2}}=\frac{d \sigma}{d K_{t}{ }^{2}}$ as equation (18) reveals. In Fig. 4, we compare the 
distributions of the final charged lepton by keeping the energy, of the incident lepton beam fixed at $100 \mathrm{GeV}$ and taking the mass of the heary lepton $m_{L}=$ 2,5,8 and $11 \mathrm{GeV}$. In Fig. 5, $\mathrm{m}_{\mathrm{L}}$ is held fixed and we take the incident lepton energy $E=50,100,200$ and $500 \mathrm{GeV}$.

Figures 6 and 7: Here the incident particle is taken to be $\overline{\mathcal{V}}$ or $l_{\mathrm{RH}}^{+}$producing $\mathrm{L}^{+}$ or $\bar{L}^{0}$ and decaying via $L^{+} \rightarrow \bar{v}(K)+\ell^{+}(Q)+\nu_{l}(\bar{k})$ or $\overline{L^{0}} \rightarrow$ $l^{+}(K)+l^{-}(Q)+\bar{\nu}_{l^{\prime}}(\bar{k}) \cdot$ Again $(V-A)$ coupling is assumed so that $\frac{d \sigma}{d Q_{t}^{2}}=\frac{d \sigma}{d K_{t}^{2}}$

Figures 8 and 9: These are the normalized versions of Figs. 4 and 5. Since, for $(V-A)$ coupling of $L, \frac{d \sigma}{d Q_{t}^{2}}=\frac{8}{m_{L}^{2}} \sigma_{p} R$. such distributions are essentially normalized to the total cross section for a fixed value of $m_{L}$ and are free of the value of $R$, unlike Figs. 4 and 5 , and are, therefore, more readily comparable to an experimental graph of $\left(\frac{\text { number of events with } Q_{t}^{2}}{\text { number of events with } Q_{t}^{2}=0}\right)$ versus $Q_{t}^{2}$.

Figures 10 and 11: Here $(V+A)$ coupling for $L$ is assumed, so the incident lepton is right handed and $\frac{d \sigma}{d Q_{f}{ }^{2}} \neq \frac{d \sigma}{d K_{t}{ }^{2}}$. The two charged leptons in the final state of decay of $L^{0}$ have significantly difierent distributions. In Fig. 10 we compare $\frac{d \sigma}{d Q_{t}^{2}}$ and $\frac{d \sigma}{d K_{t}^{2}}$ for fixed energy $E=100 \mathrm{GeV}$ and for $m_{L}=2$ and $8 \mathrm{GeV}$. In Fig. 11, we hold $m_{L}=5 \mathrm{GeV}$ fixed and compare $\frac{d \sigma}{d Q_{t}^{2}}$ and $\frac{d \sigma}{d K_{t}^{2}}$ for $E=50,200$ and $500 \mathrm{GeV}$.

Figures 12 and 13: These are for $(V+A)$ coupling assuming a left handed incident antilepton. Again in Fig. 12, we compare $\frac{d \sigma}{d Q_{t}{ }^{2}}$ and $\frac{d \sigma}{d K_{t}{ }^{2}}$ for fixed $E$ and 
for $m_{L}=2$, and $8 \mathrm{GeV}$. In Fig. $13, m_{L}$ is held fixed at $5 \mathrm{GeV}$ and $E$ is taken to be 50,200 and $500 . \mathrm{GeV}$.

Figures 14 and 15: These figures show the polarization effects of $L$ in the reaction

$$
\begin{aligned}
\nu(k)+N(p) & \longrightarrow L^{-}\left(k^{2}\right)+\text { " anything" } \\
& \longrightarrow \nu(K)+l^{-}(Q)+\bar{\nu}_{l}(\bar{k})
\end{aligned}
$$

(V-A) coupling for $L$ is assumed. In Fig. 14, we plot the degreee of left handedness of $L$, defined by $P_{L H}=\frac{1-\hat{S} \cdot \hat{k}^{b}}{2} \times 100 \%$, where $\hat{s}$ is the polarization unit vecior in the rest frame of $L$ and $\hat{k}^{\prime}$ is the 3-momentum unit vector of $L$ in the lab frame, versus $k_{0}^{0}$ which is the energy of the heavy lepton in the lab frame, keeping the scattering angle . $\theta^{\prime}$ fixed. The effects of the polarization of $L$ on the spectrum of the final lepton under observation is shown in Fig. 15. The figure shows the corrections, $D_{0}$ and $D_{L}$, defined in equations (37) and (38), as a function of the (transverse momentum) $)^{2}$ for two different heavy lepton masses $m_{L}=5$ and $8 \mathrm{GeV}$ with $E=100 \mathrm{GeV}$. 


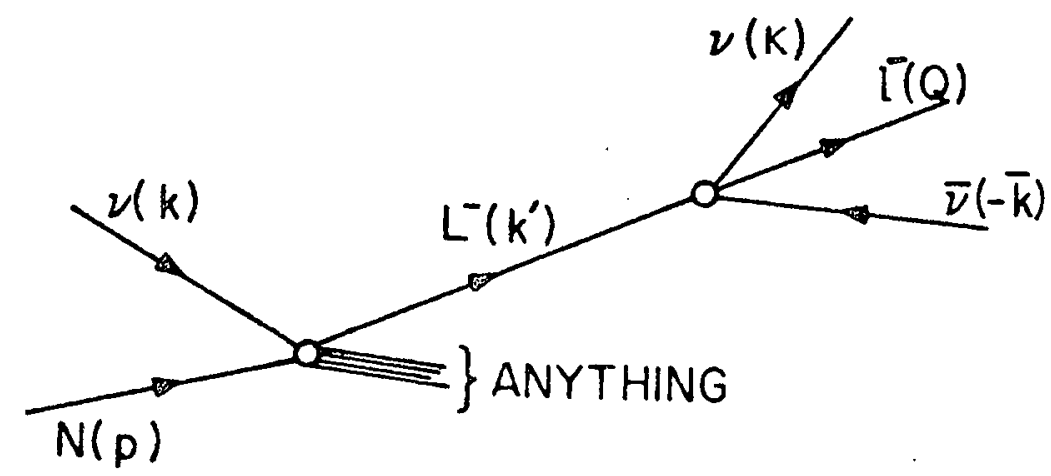

Figure 1. 


$$
\text { 为 }
$$




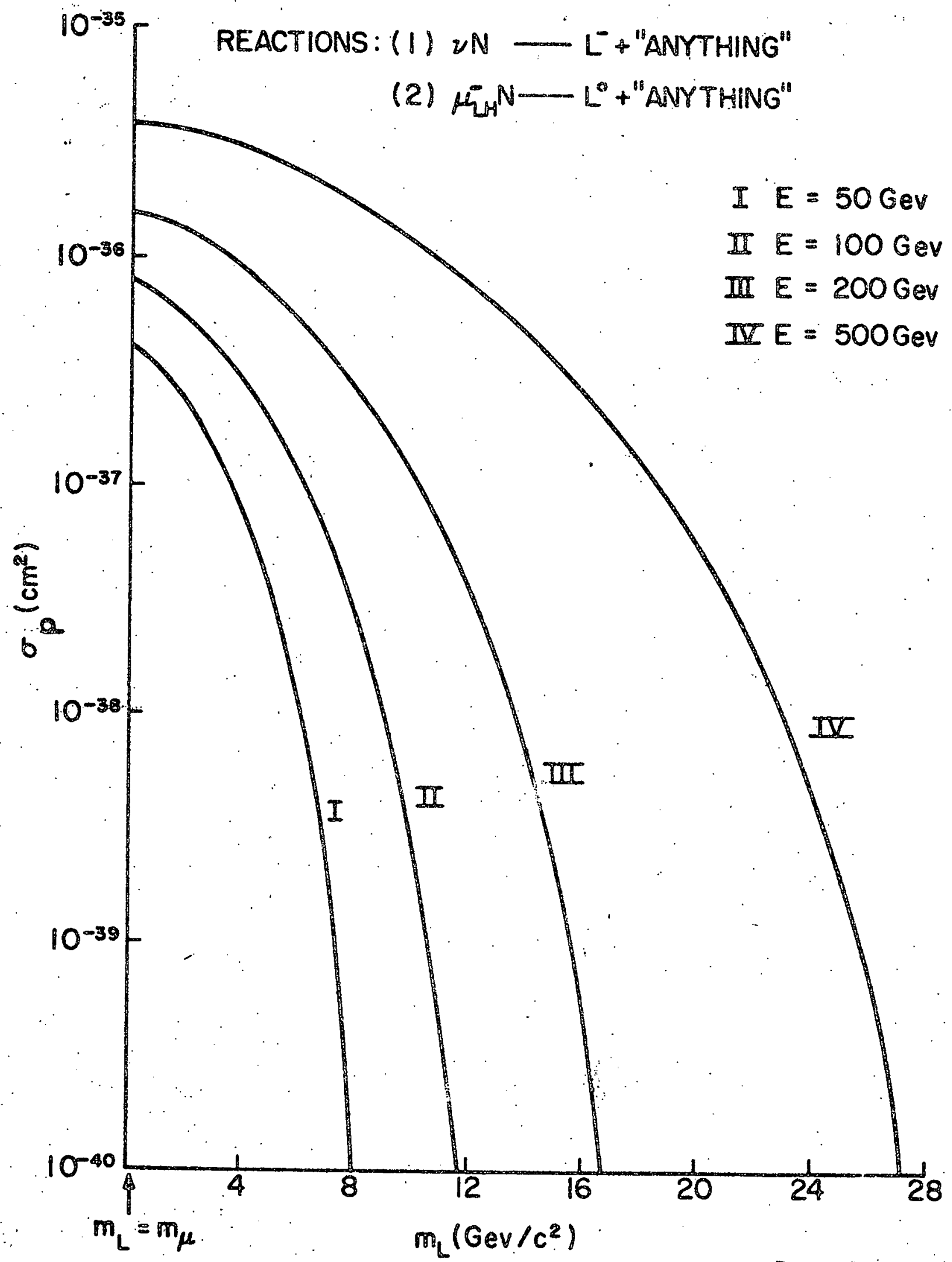

Figure 3. 


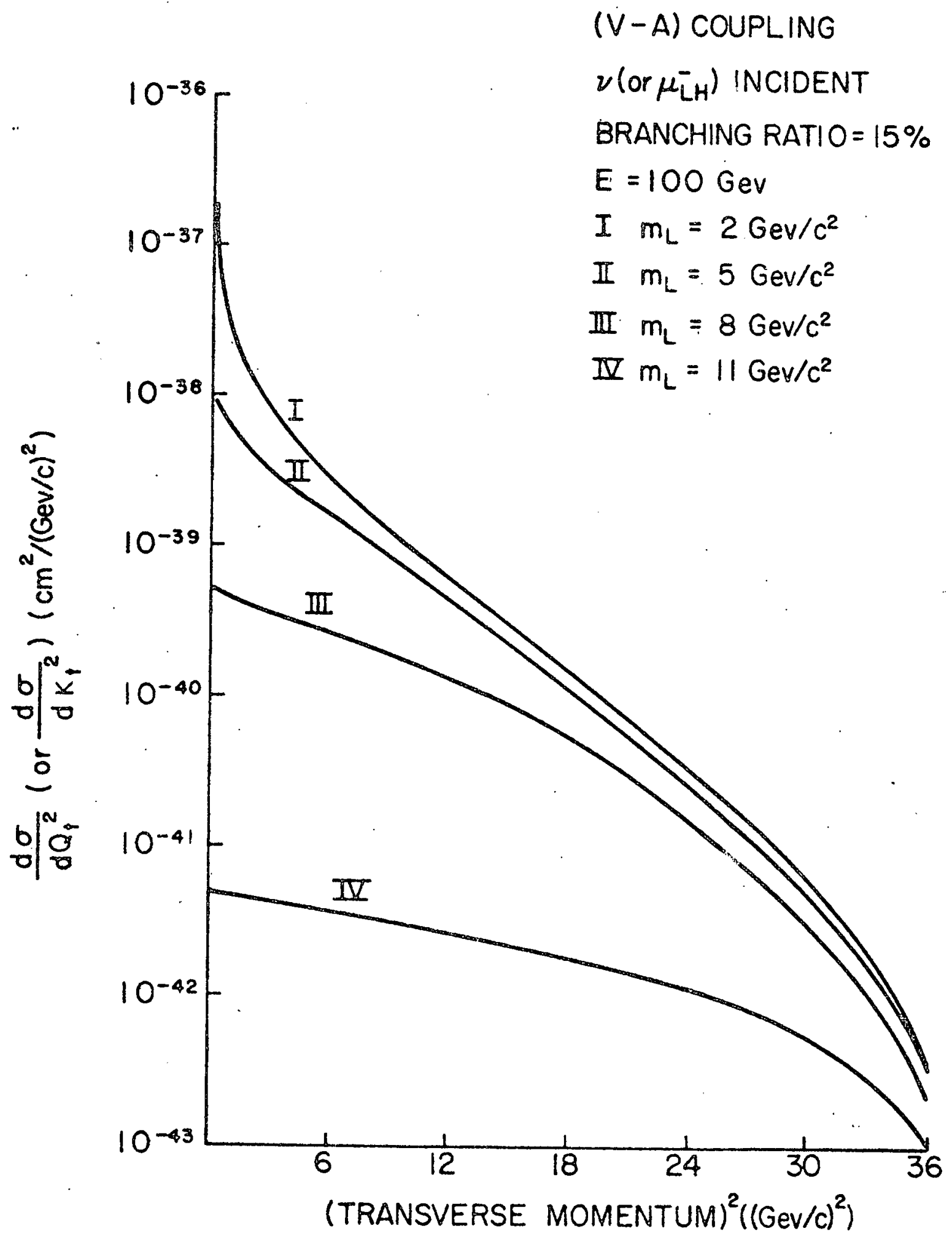

Figure 4. 


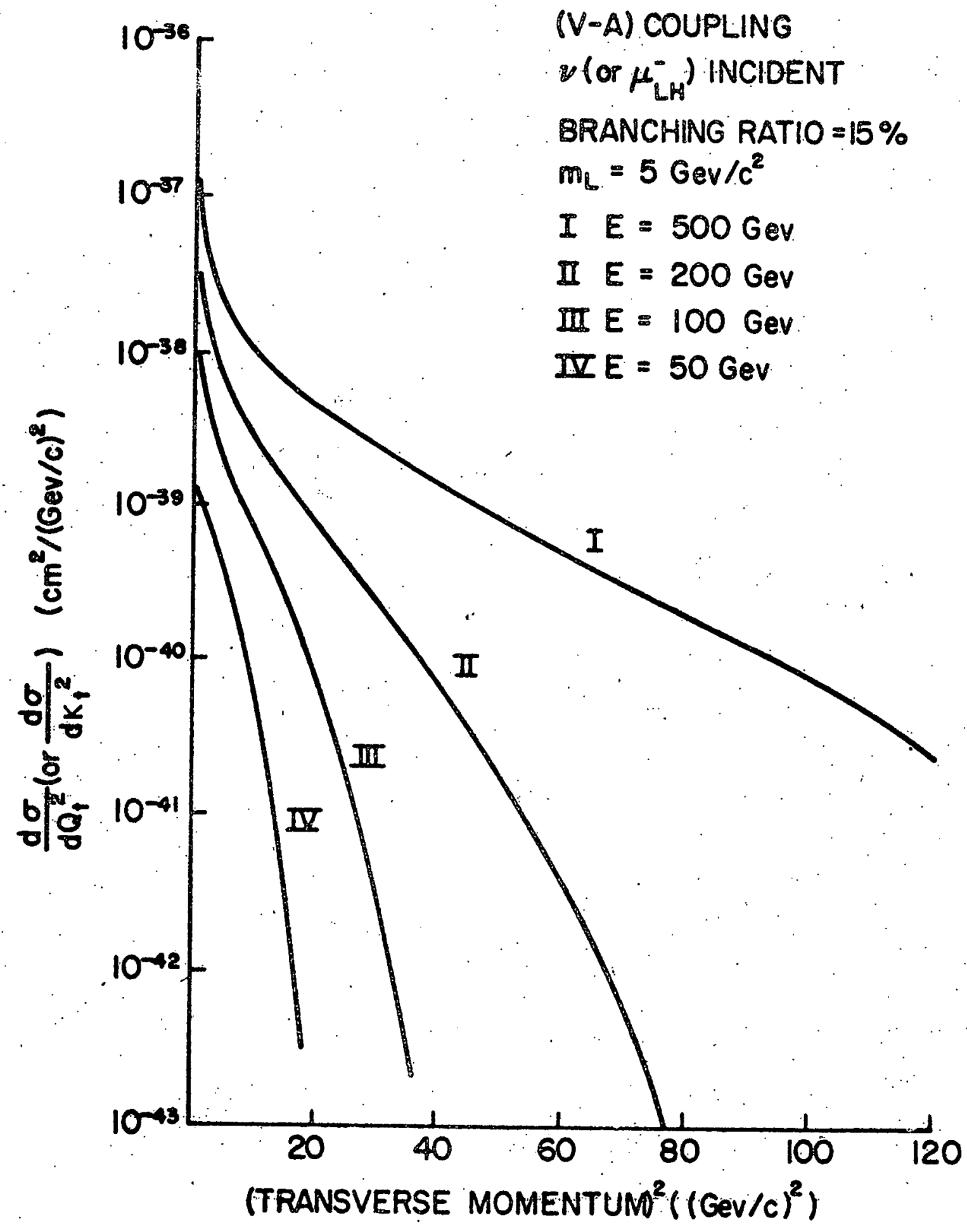

Figure 5. 


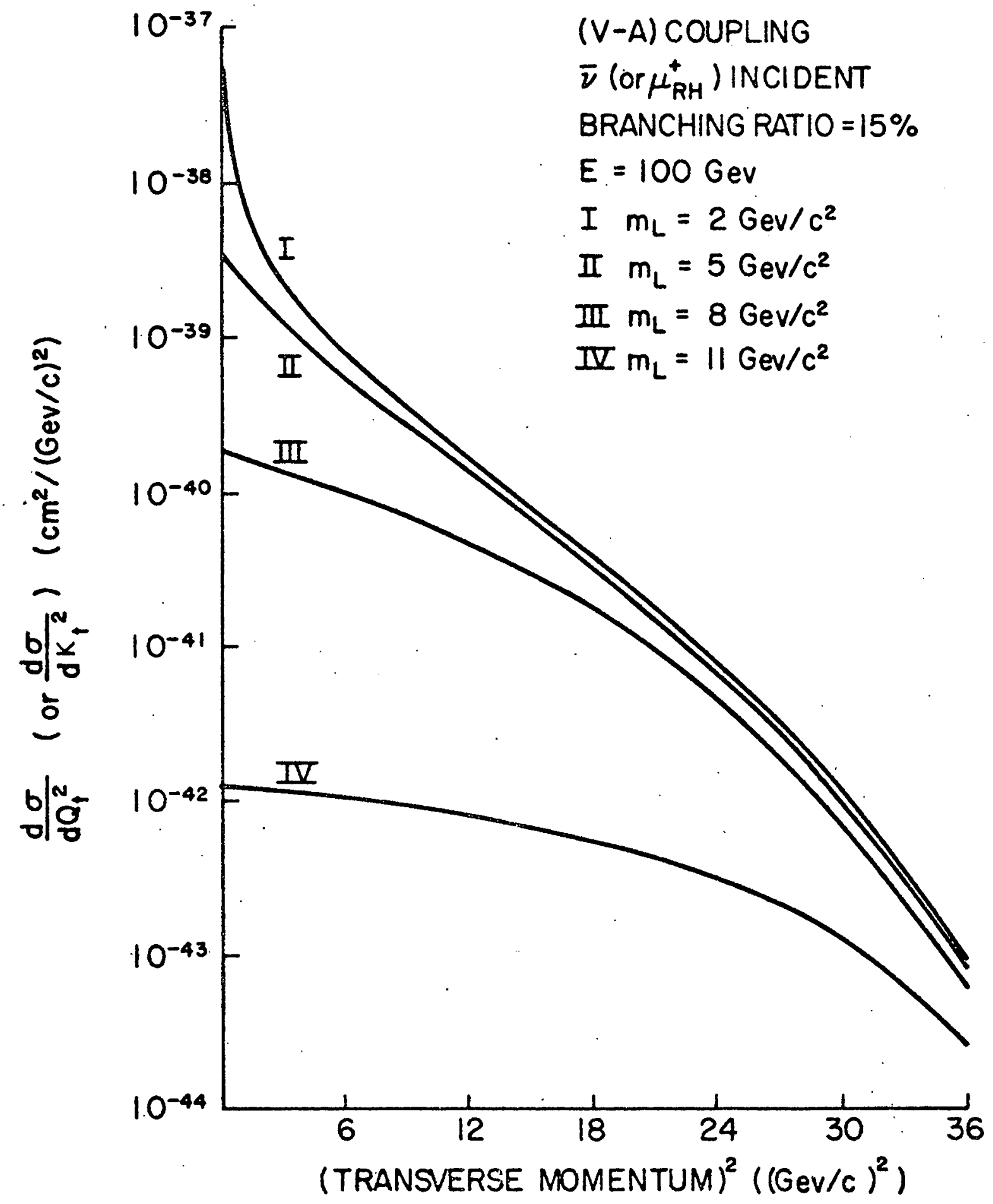

Figure 6. 


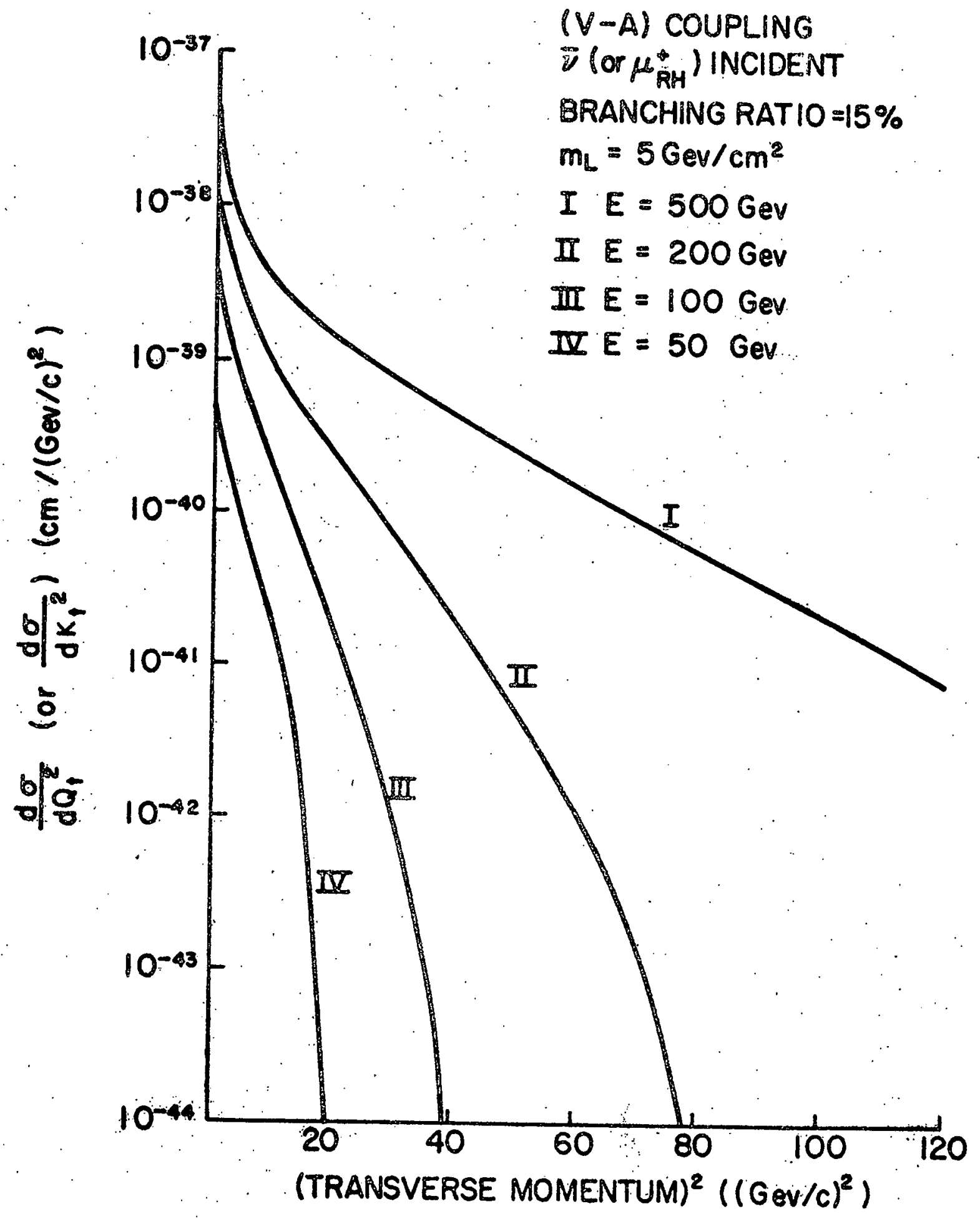

Figure 7. 
$(V-A)$ COUPLING, $\nu$ (or $\mu_{L H}^{-}$) INCIDENT.

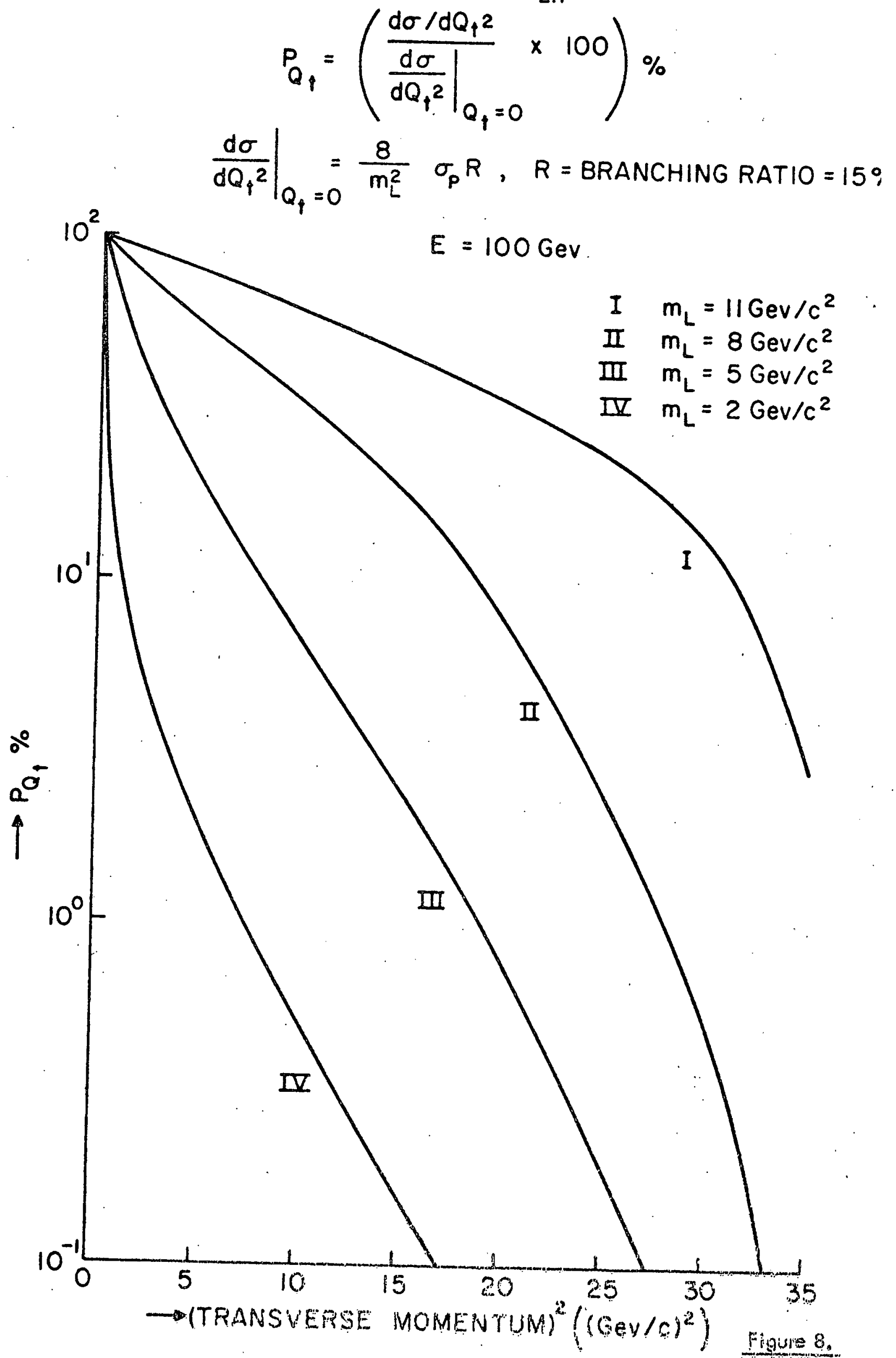


$(V-A)$ COUPLING, $\nu$ (or $\mu_{L H}^{-}$) INCIDENT.

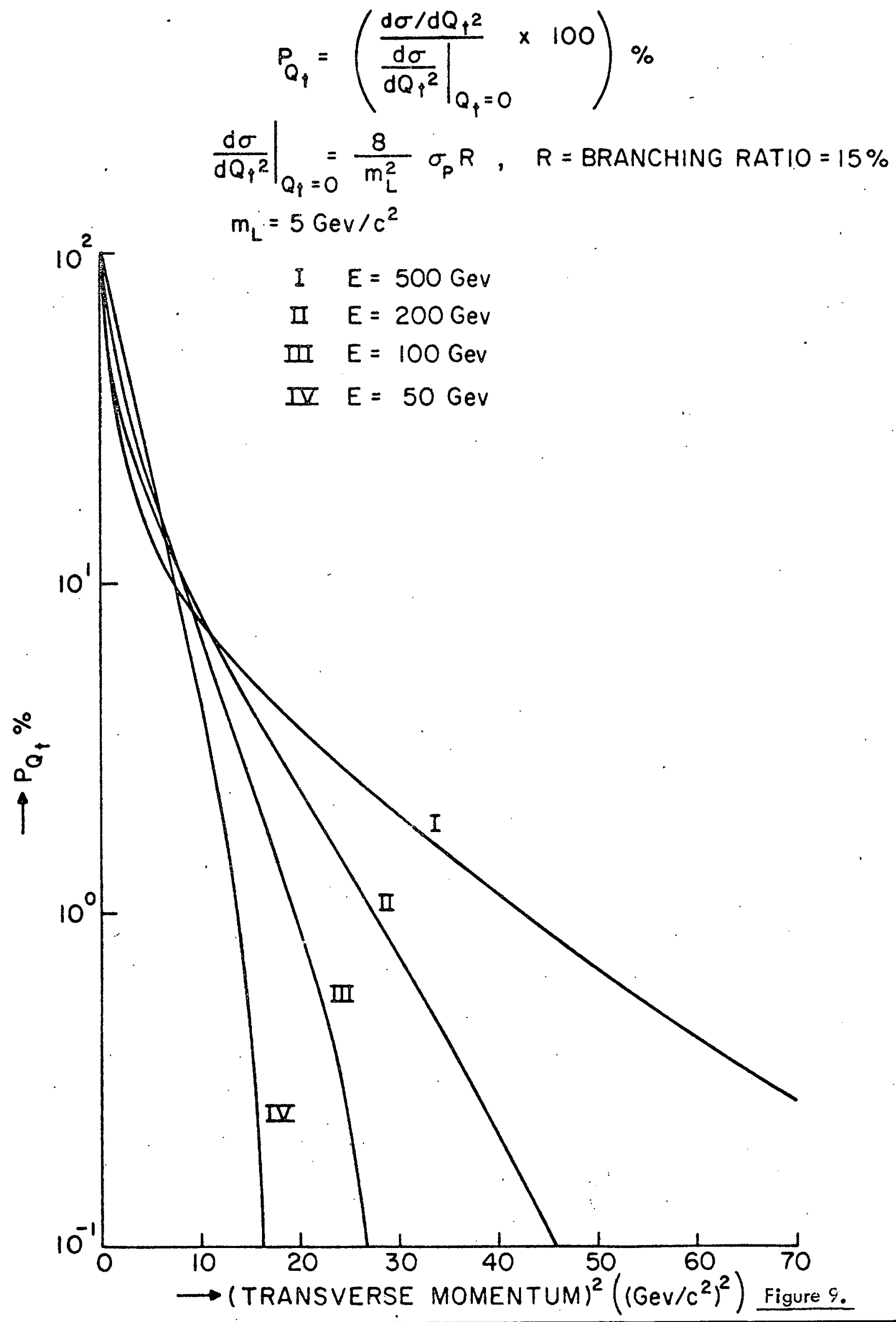




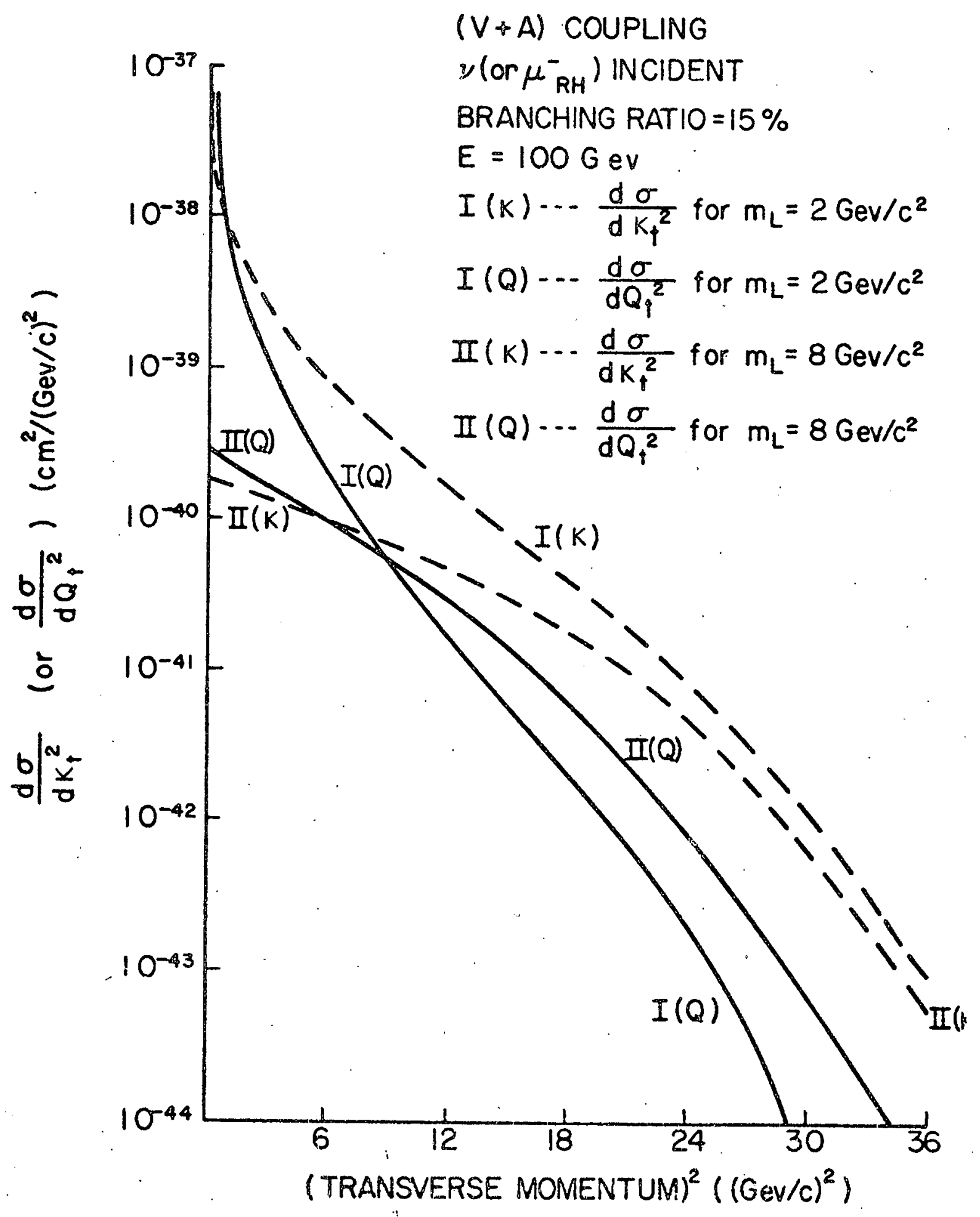

Figure 10. 


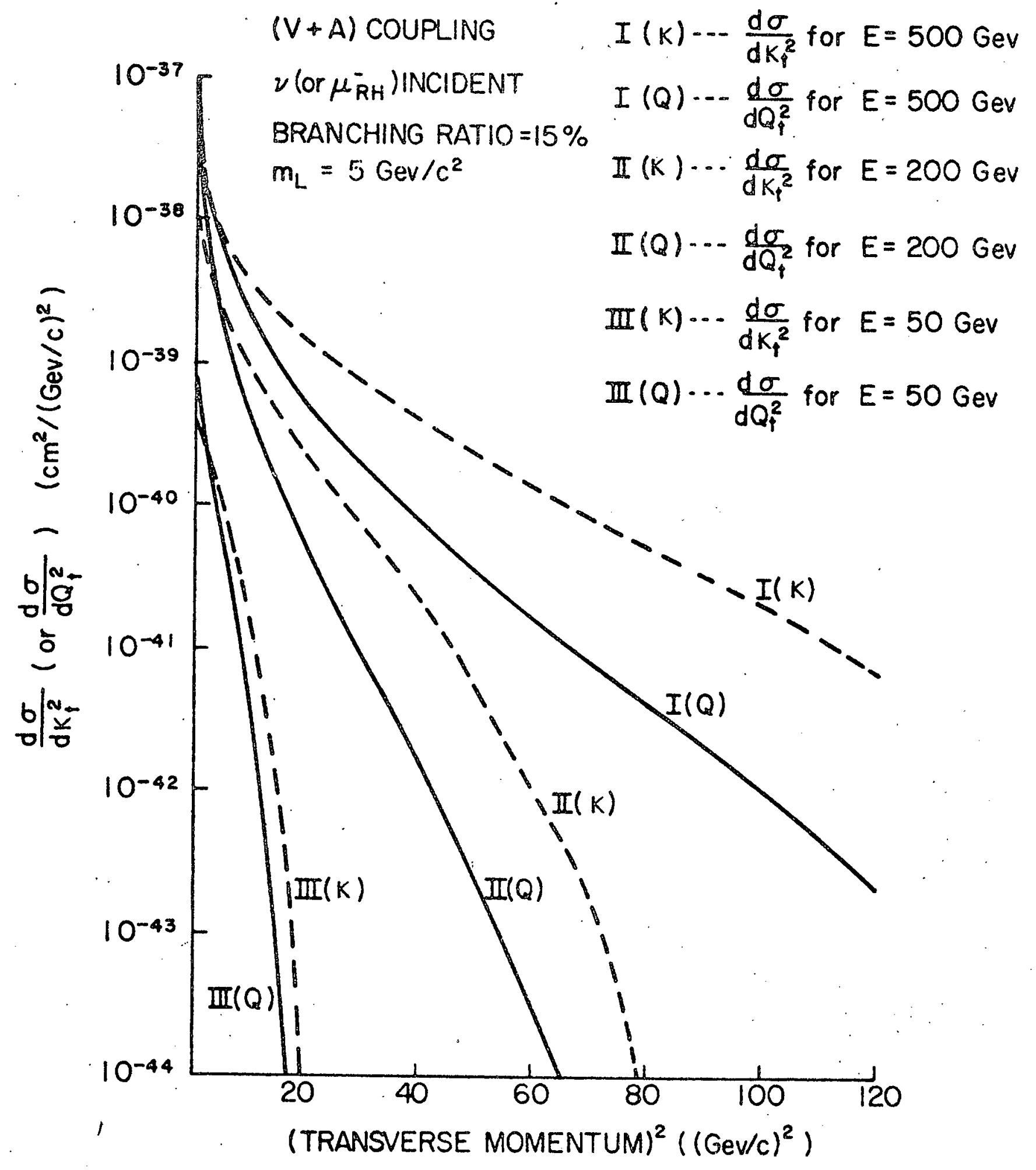

Figure 11. 


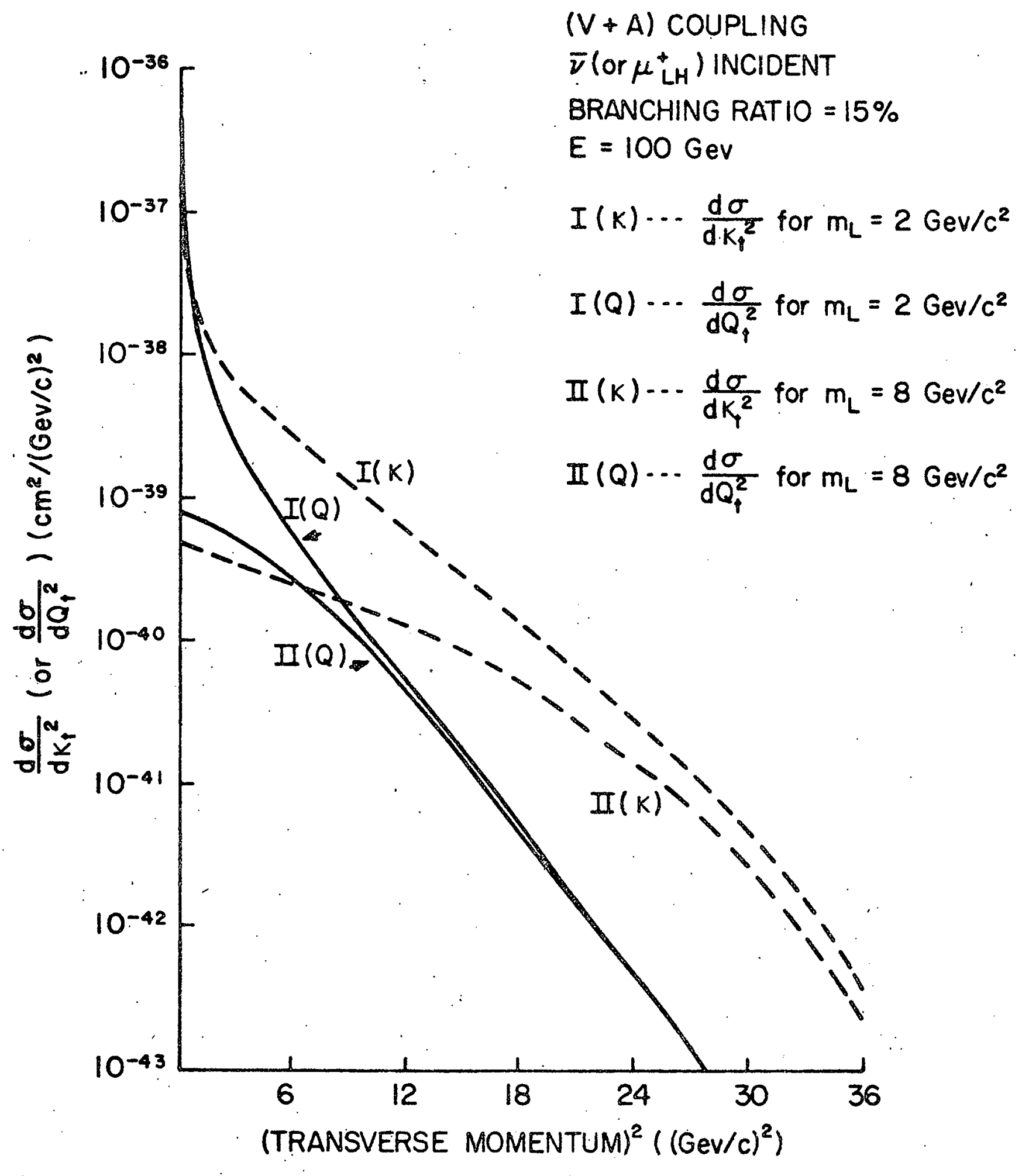

Figure 12. 


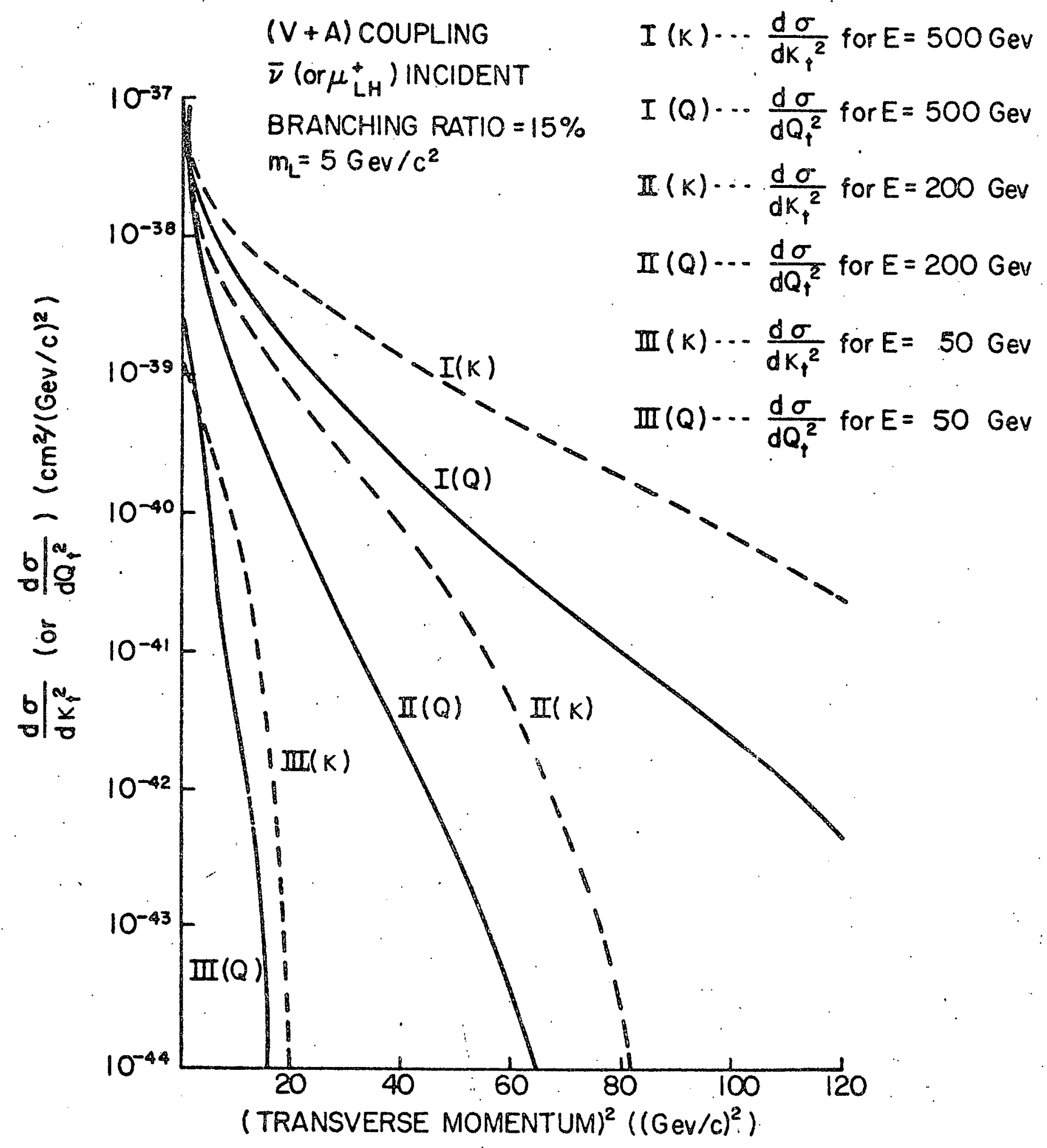

Figure 13. 


$$
\begin{gathered}
P_{L H}=\frac{1-\hat{s} \cdot \hat{k}^{\prime}}{2} \\
E=100 \mathrm{Gev}\left\{\begin{array}{cc}
I \cdots m_{L}=5 \mathrm{Gev} / c^{2} & \theta^{\prime}=2^{\circ} \\
\text { II - } m_{L}=5 \mathrm{Gev} / c^{2} & \theta^{\prime}=5^{\circ} \\
\text { III } \cdots m_{L}=8 \mathrm{Gev} / \mathrm{c}^{2} & \theta^{\prime}=2^{\circ}
\end{array}\right. \\
E=200 \mathrm{Gev} \text { III } \cdots m_{L}=5 \mathrm{Gev} / \mathrm{c}^{2} \quad \theta^{\prime}=2^{\circ}
\end{gathered}
$$

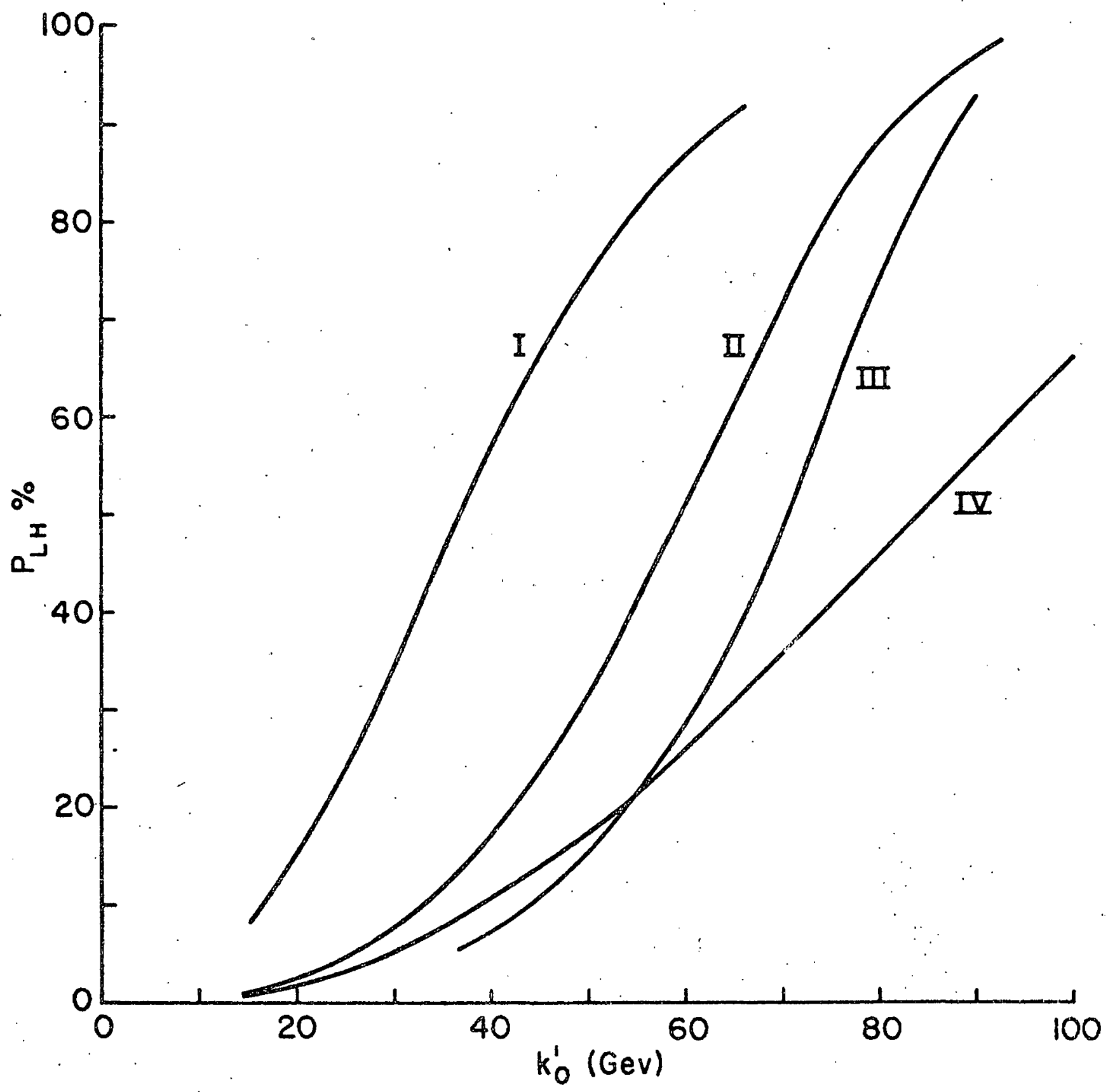

Figure 14. 


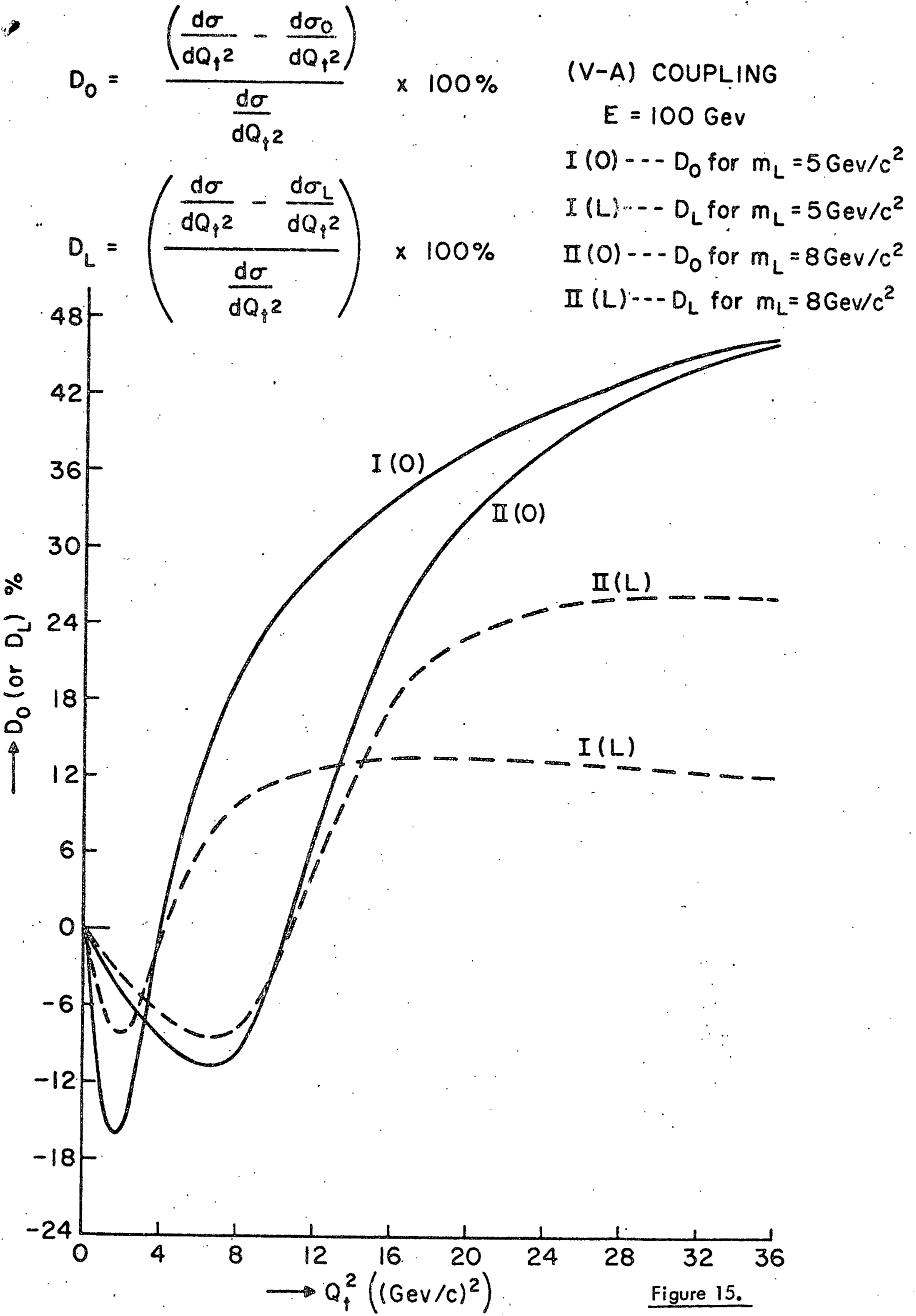

